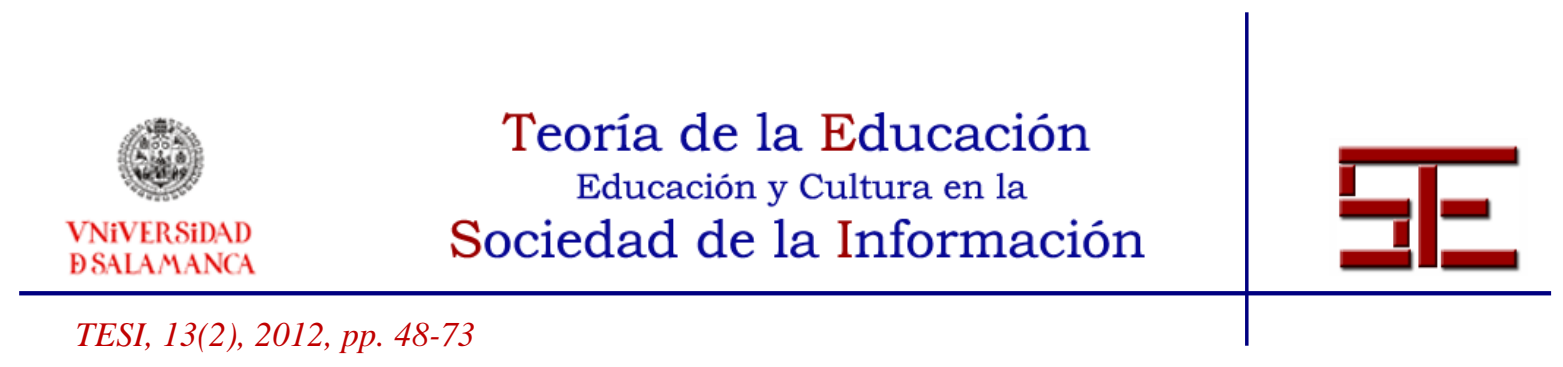

\title{
SIMULACIÓN ROBÓTICA CON HERRAMIENTAS 2.0 PARA EL DESARROLLO DE COMPETENCIAS BÁSICAS EN ESO. UN ESTUDIO DE CASOS
}

Resumen: El artículo presenta una investigación contextualizada en un centro de enseñanza secundaria de la provincia de Toledo en el que se analiza el diseño de una actividad de simulación robótica para el desarrollo de competencias básicas de forma transversal e interdisciplinar entre las materias de Tecnología, Lengua española, Lengua inglesa y Educación Plástica y Visual. El diseño de la actividad consistió en el desarrollo de un proyecto interdisciplinar para la construcción de un robot gusano con un dispositivo electrónico que lo convierte en un robot para medir longitudes. Para su diseño y desarrollo se utilizaron cuatro herramientas 2.0 gratuitas que sirvieron de soporte al desarrollo de competencias básicas: Google Sketchup, Prezi, Glosgter y Windows Movie Maker. Se analizaron las apreciaciones de docentes y alumnos sobre la viabilidad de la simulación robótica a través del trabajo por proyectos como estrategia didáctica y especialmente la mejora de indicadores de las siguientes competencias básicas: lingüística, aprender a aprender, tratamiento de la información y competencia digital y conocimiento y la interacción con el mundo físico. Los resultados obtenidos muestran una alta apreciación de alumnado y profesorado sobre el proyecto y su funcionalidad en el desarrollo transversal e interdisciplinar de las competencias básicas.

Palabras clave: Robótica educativa; TIC; competencias básicas; trabajo por proyectos; innovación curricular.

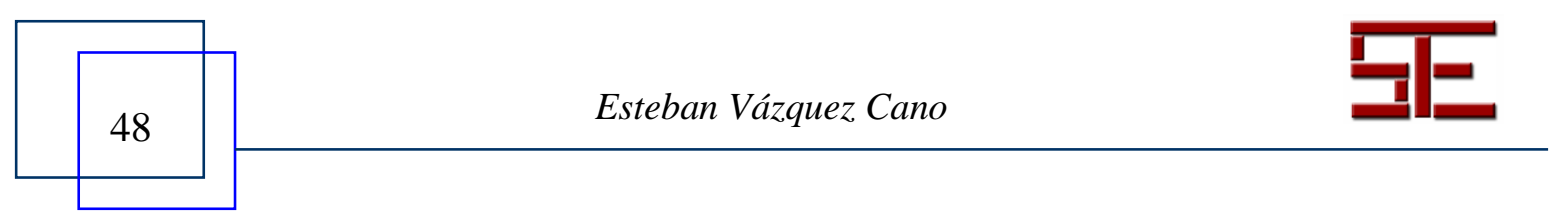




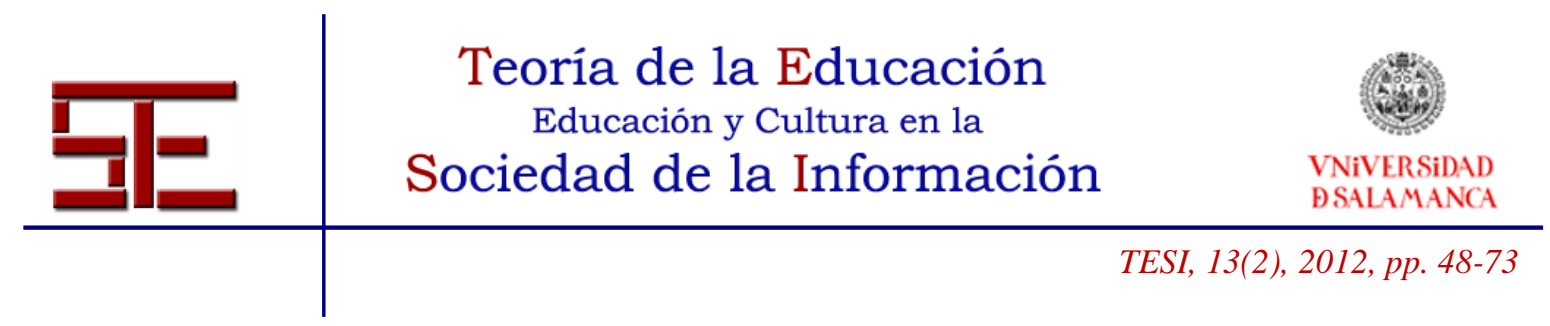

\title{
ROBOTIC SIMULATION WITH 2.0 TOOLS TO DEVELOP KEY COMPETENCIES IN SECONDARY EDUCATION. A CASE STUDY
}

\begin{abstract}
The paper presents a contextualized research in a secondary school in the province of Toledo in which is examined the design of a robotic simulation activity for the development of key competencies from an interdisciplinary and transversely way between the subjects of Technology, Spanish Language, English and Plastic and Visual Education. The design of the activity consisted on the development of an interdisciplinary project for the construction of a worm robot with an electronic device for measuring lengths. 2.0 free tools were used for designing it and supported the development of four key competencies: Google Sketchup, Prezi, Glosgter and Windows Movie Maker. We analyzed the findings of teachers and students on the feasibility of robotic simulation through the project work as a teaching strategy and especially the improvement of indicators of the following basic skills: language, learning to learn, information processing and digital competence knowledge and interaction with the physical world. The results show a high appreciation of students and teachers about the project and its functionality in the cross and interdisciplinary development of key competencies.
\end{abstract}

Keywords: Educational robotics; ICT; key competencies; project work; curriculum innovation.

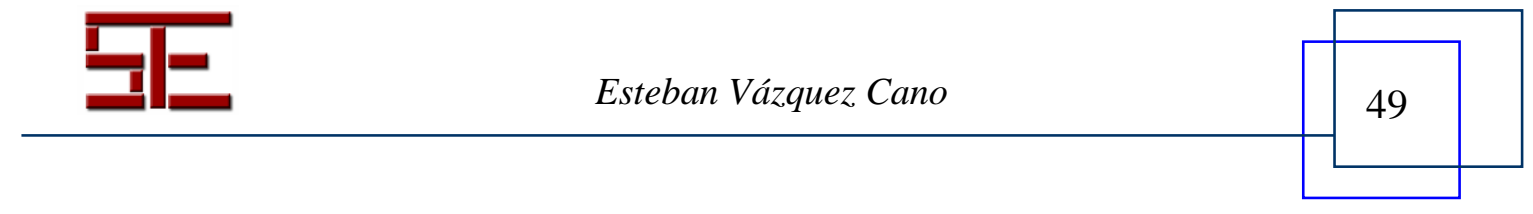




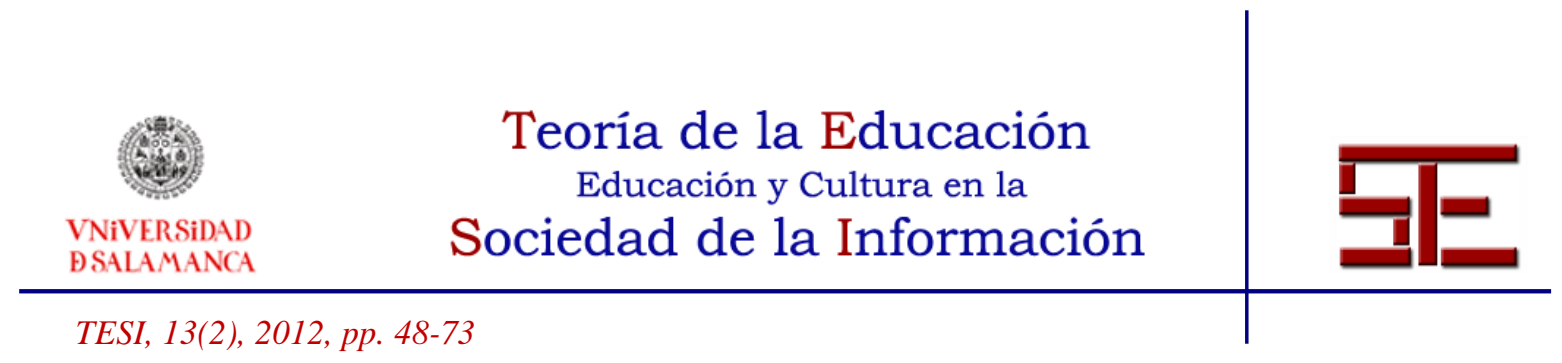

\section{SIMULACIÓN ROBÓTICA CON HERRAMIENTAS 2.0 PARA EL DESARROLLO DE COMPETENCIAS BÁSICAS EN ESO. UN ESTUDIO DE CASOS}

Fecha de recepción: 17/05/2012; fecha de aceptación: 02/07/2012; fecha de publicación: 26/07/2012

Esteban Vázquez Cano

evazquez@edu.uned.es

UNED

\section{1.- INTRODUCCIÓN}

El artículo presenta una investigación contextualizada en el segundo curso de la Enseñanza Secundaria Obligatoria en la que se analiza el diseño de una actividad de simulación robótica para el desarrollo de competencias básicas de forma transversal e interdisciplinar entre las materias de Tecnología, Lengua española, Lengua inglesa y Educación Plástica y Visual en un centro de enseñanza secundaria de la provincia de Toledo. El diseño didáctico aplicado ha sido el método de proyectos a partir de un desarrollo transversal e interdisciplinar de objetivos y contenidos planteados de forma que su puesta en práctica supusiera un desarrollo funcional de unas destrezas enmarcadas en los indicadores de desarrollo de las competencias básicas. Para su ejecución, se han empleado cuatro herramientas 2.0 gratuitas que suponen un manejo asociado de destrezas aplicables de forma interdisciplinar en las cuatro materias implicadas. Las herramientas 2.0 utilizadas han sido: Google Sketchup para el diseño digital del robot, Glogster para realizar un póster interactivo en el que explicar los pasos y divulgar la experiencia en inglés, Prezi para el diseño de una presentación interactiva que sirva de soporte electrónico a la explicación oral de la experiencia en la materia de lengua castellana y Windows Movie Maker para el desarrollo de un vídeo del proceso que sirviera como videosimulación de lo desarrollado y aprendido.

El trabajo por competencias en la dinámica de enseñanza-aprendizaje propugnada a nivel europeo y español establece ocho competencias básicas que el alumno debe adquirir al finalizar la enseñanza secundaria (OECD, 2002; Parlamento Europeo, 2006; MEC, 2006; LOE, 2006). La didáctica y evaluación del desarrollo de estas competencias es todavía una asignatura pendiente del sistema educativo español. Para el desarrollo de didácticas con base en las competencias básicas el mismo Parlamento Europeo establece que hay una serie de temas que se aplican a lo largo del marco de referencia y que intervienen en las ocho competencias clave: el pensamiento crítico, la creatividad, la capacidad de iniciativa, la resolución de problemas, la evaluación del

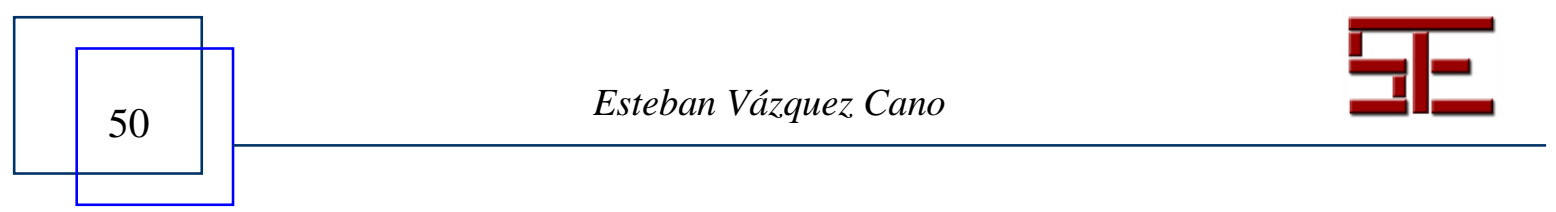




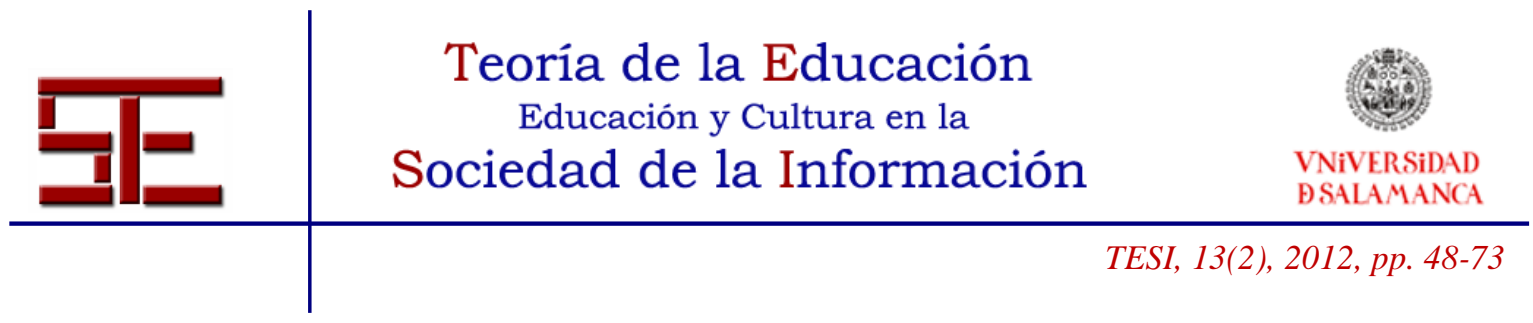

riesgo, la toma de decisiones y la gestión constructiva de los sentimientos (L394/142006/962/CE). Asimismo, la LOE, 2006, en su Anexo I, establece que con las áreas y materias del currículo se pretende que los alumnos y las alumnas alcancen los objetivos educativos y, consecuentemente, también que adquieran las competencias básicas. Sin embargo, no existe una relación unívoca entre la enseñanza de determinadas áreas o materias y el desarrollo de ciertas competencias. Cada una de las áreas contribuye al desarrollo de diferentes competencias y, a su vez, cada una de las competencias básicas se alcanzará como consecuencia del trabajo en varias áreas o materias. A partir de estas consideraciones, planteamos esta investigación para analizar la potencialidad didáctica en el desarrollo de competencias básicas a través del trabajo por proyectos con base en la robótica educativa y con el apoyo de herramientas 2.0.

\section{1.- Objetivos de la investigación}

La investigación se ha estructurado para dar respuesta a los siguientes objetivos:

1. Describir la actividad de simulación robótica como parte de un proceso de innovación curricular.

2. Analizar las apreciaciones de los sujetos implicados (profesorado y alumnado) en el desarrollo de la actividad.

3. Comprobar la funcionalidad de las herramientas 2.0 en el desarrollo de proyectos de robótica educativa.

4. Analizar la funcionalidad de los proyectos interdisciplinares con base en la robótica educativa y las TIC en el desarrollo de las competencias básicas.

\section{2.- MARCO TEÓRICO}

La robótica educativa en la actualidad dentro del contexto escolar enfocado al desarrollo de competencias básicas puede ser una excelente estrategia didáctica complementada con el apoyo de las tecnologías digitales. Su uso y aplicabilidad se extiende desde los primeros cursos de primaria hasta las enseñanzas universitarias (Mataric, 2004; Blank, Kumar, Meeden \& Yanco, 2004). Su aplicación a la práctica docente lleva aparejado que el alumnado simule proyectos de aplicabilidad real y funcional en tareas del día a día, profesionales, académicas o vivenciales y supone un uso contextualizado de códigos gráficos, textuales, visuales y lingüísticos. Muestra de su potencialidad en la enseñanza son proyectos europeos como: "Teacher Education on Robotics-Enhanced Constructivist Pedagogical Methods-TERECoP", que se ha desarrollado durante los años 2006-2009 con la participación de seis países europeos (www.terecop.eu).

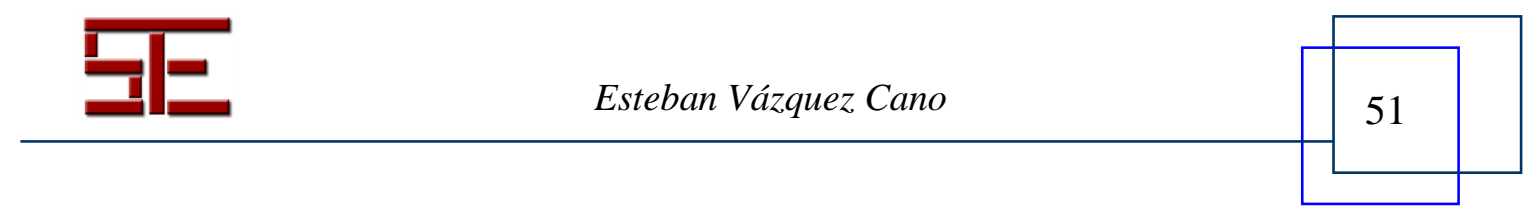




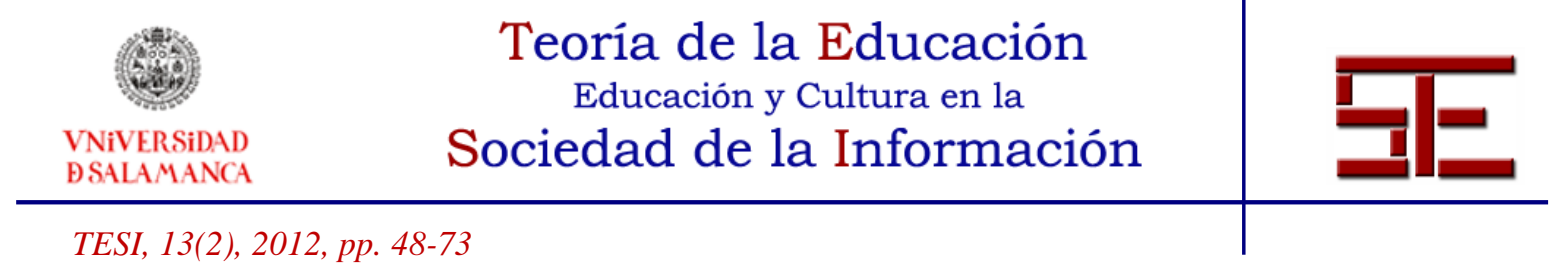

En la actualidad, la robótica educativa es un enfoque didáctico que ofrece funcionalidad y aplicabilidad al alumnado (Resnick, Martin, Sargent \& Silverman, 1996; Miglino, Lund \& Cardaci, 1999; Denis \& Hubert, 2001; Sklar \& Eguchi, 2004). La investigación actual sobre robótica educativa se orienta principalmente hacia tres campos de actuación; uno primero se enfoca hacia el uso de las tecnologías en el desarrollo de proyectos educativos con base en la robótica educativa (Mor, Hoyles, Kahn, Noss \& Simpson, 2006; Resnick, 2006). Otra línea de investigación es la búsqueda de la aplicabilidad y funcionalidad de los constructos en el contexto mediato del estudiante (Breazeal, 2002; Kumar, 2004; Ionita \& Ionita, 2007); y junto a estos dos aproximaciones, aparecen los procesos de comunicación insertos en estos proyectos como medio de interacción, reflexión y pensamiento crítico en el desarrollo de sus fases (Thomas, Mergendoller \& Michaelson, 1999; Han \& Bhattacharya, 2001).

Tomando como referencia estas tres aproximaciones teóricas, el proyecto que presentamos contempla de forma interrelacionada los principios de funcionalidad de las herramientas 2.0 en el desarrollo de proyectos interdisciplinares con base en la robótica educativa. Parte de la funcionalidad del proyecto y su aplicabilidad en un contexto cercano y real para el alumno, para lo que se inserta en la dinámica de trabajo metodológico con base en el desarrollo de las competencias básicas; y, por último, aplica los principios de organización y comunicación digital de los resultados obtenidos. Esta aproximación teórica que podemos denominar como integrada, la podemos encontrar parcialmente en (Jones \& Flynn, 1993; Penner, 2001; Weiss, 2008). Para el desarrollo de la robótica educativa, uno de los modelos de intervención pedagógica con más éxito y el empleado en esta experiencia didáctica es el método de proyectos; ya que supone un trabajo colaborativo y cooperativo que fomenta el pensamiento crítico, la evaluación de opciones y la resolución de problemas desde un enfoque creativo y constructivista (Bransford \& Stein, 1993). El método por proyectos se basa en tareas complejas que desarrollan diferentes destrezas de forma reflexiva y funcional (Blumenfeld et al., 1991; Knoll, 1997). El trabajo por proyectos es una aproximación sólida para el desarrollo de programas educacionales de robótica educativa y de competencias básicas al intentar transferir los resultados en una práctica real o funcional fuera del aula. Además supone una oportunidad única para que el profesorado y alumnado trabaje en grupo y cambie una didáctica más tradicional por otra más colaborativa y facilitadora del (co)aprendizaje (Han \& Bhattacharya, 2001). De esta forma, se avanza hacia una didáctica denominada aprender diseñando (Gagnon \& Collay, 2001). Esta didáctica resalta el valor de aprender creando, programando y

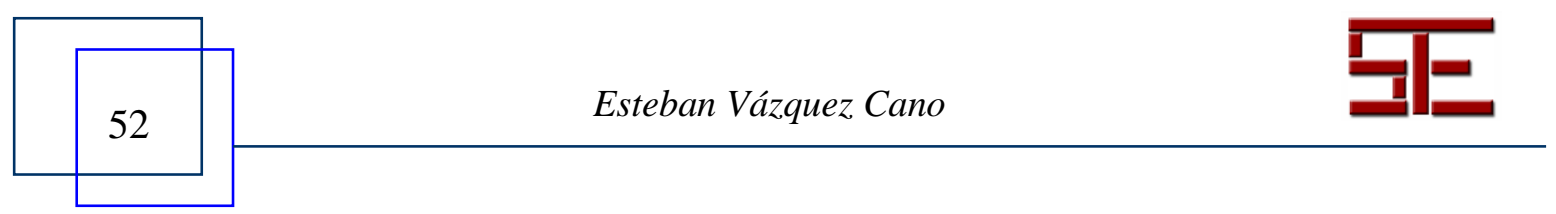




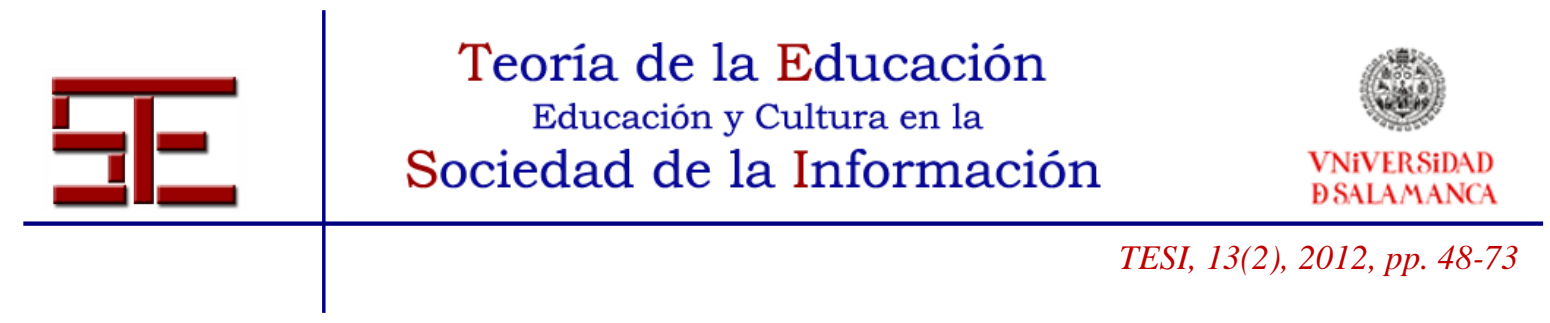

participando en el diseño de un producto funcional, práctico y significativo para el discente. Diseñar un robot supone un proceso complejo en el que se ven implicadas la creatividad y la habilidad para resolver los problemas que se van generando (Druin \& Hendler, 2000; Erstad, 2002; Carbonaro, Sklar, Parsons \& Azhar, 2007; Puntambekar, \& Kolodner, 2005), y en el desarrollo y ejecución de este proceso es fundamental el trabajo por competencias. Mediante actividades concretas y el apoyo de las TIC, los estudiantes ponen en juego diversos conocimientos, habilidades, destrezas y actitudes relacionadas con el trabajo colaborativo (Oliver, 2002; Lipponen, 2003; Cabero, 2003). El alumnado trabaja en equipo para conseguir el desarrollo de un producto que será compartido y valorado por terceros y que le guiará a través de la comprensión de conceptos relevantes en las diferentes materias implicadas (Penner, 2001; Coll, 2001). Asimismo, García-Valcárcel (2009) afirma que al involucrar a los estudiantes en una tarea con el propósito de resolver un problema o elaborar un producto específico, se potencian las habilidades intelectuales y se promueve la responsabilidad personal y de equipo. En el desarrollo de este método por proyectos con base en la robótica aparecen las herramientas 2.0 como poderosos recursos facilitadores (Vázquez Cano \& Sevillano García, 2011). Para el desarrollo interdisciplinar de trabajos por proyectos se deben utilizar herramientas 2.0 de acceso libre y fácil uso entre el profesorado y el alumnado; de esta manera se potencia el uso transversal y contextualizado de contenidos.

\section{3.- DESCRIPCIÓN DE LA ACTIVIDAD}

La actividad se desarrolló durante el segundo trimestre del curso 2010/11 y en ella participaron profesorado y alumnado de cuatro materias de segundo curso de la ESO: Taller Tecnológico, Lengua española, Lengua inglesa y Educación Plástica y Visual. El objetivo de interrelacionar cuatro materias diferentes del currículo responde a dos principios didácticos:

1. Trabajo transversal e interdisciplinar de objetivos generales de la etapa de secundaria. Principalmente los reseñados en la Tabla 1.

Tabla 1. Objetivos de etapa implicados en el proyecto. (LOE. Art. 23)

\begin{tabular}{l}
\hline Objetivos Etapa. $L O E$ - art. 23 \\
\hline e) Desarrollar destrezas básicas en la utilización de las fuentes de información para, con sentido \\
crítico, adquirir nuevos conocimientos. Adquirir una preparación básica en el campo de las \\
tecnologías, especialmente las de la información y la comunicación.
\end{tabular}

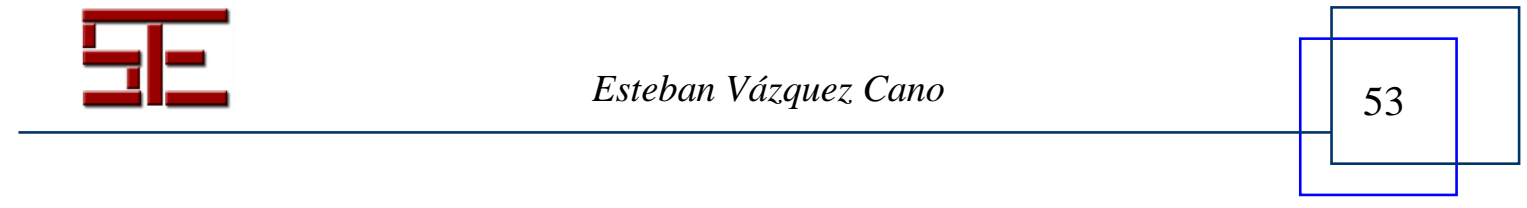




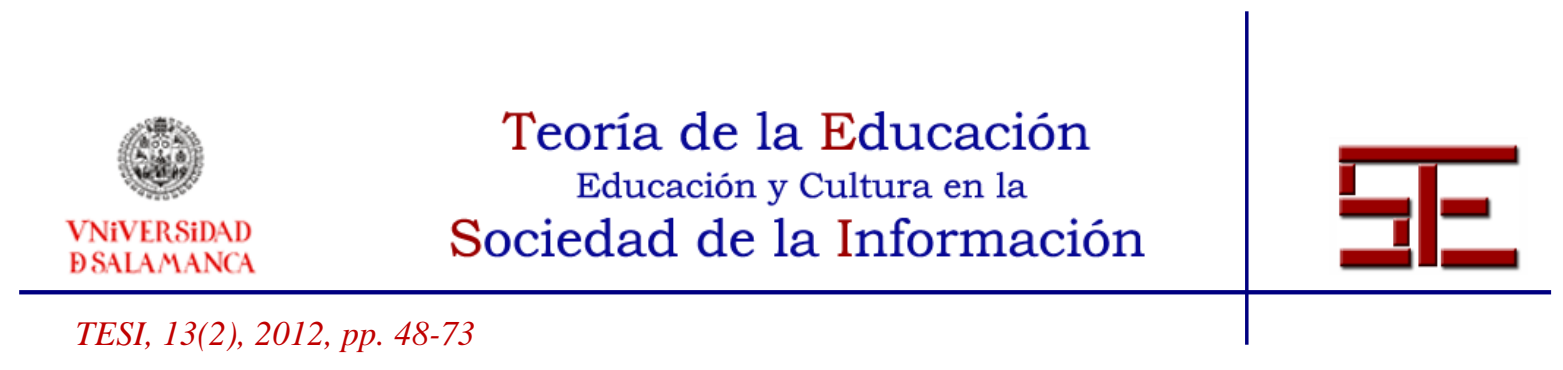

f) Concebir el conocimiento científico como un saber integrado, que se estructura en distintas disciplinas, así como conocer y aplicar los métodos para identificar los problemas en los diversos campos del conocimiento y de la experiencia.

g) Desarrollar el espíritu emprendedor y la confianza en sí mismo, la participación, el sentido crítico, la iniciativa personal y la capacidad para aprender a aprender, planificar, tomar decisiones y asumir responsabilidades.

h) Comprender y expresar con corrección, oralmente y por escrito, en la lengua castellana y, si la hubiere, en la lengua cooficial de la Comunidad Autónoma, textos y mensajes complejos, e iniciarse en el conocimiento, la lectura y el estudio de la literatura.

i) Comprender y expresarse en una o más lenguas extranjeras de manera apropiada.

j) Conocer, valorar y respetar los aspectos básicos de la cultura y la historia propias y de los demás, así como el patrimonio artístico y cultural.

2. Trabajo interdisciplinar de competencias básicas. Principalmente se trataron indicadores de las siguientes competencias básicas: lingüística, aprender a aprender, tratamiento de la información y competencia digital y conocimiento y la interacción con el mundo físico.

Se atendió a la siguiente distribución de tareas organizadas en cinco fases:

Tabla 2. Cronograma y explicación del proyecto

\begin{tabular}{|c|c|c|}
\hline Fases & Materias & Desarrollo y competencias implicadas \\
\hline $\begin{array}{l}\text { 1. Diseño digital del robot } \\
\text { con la herramienta } \\
\text { Google Sketchup. (Ver } \\
\text { Fig. 1). }\end{array}$ & $\begin{array}{l}\text { Tecnología. } \\
\text { Educación Plástica } \\
\text { y Visual. }\end{array}$ & $\begin{array}{l}\text { - El alumnado desarrolló en la materia de Educación } \\
\text { Plástica y Visual un boceto a lápiz y carboncillo de la } \\
\text { figura del robot. } \\
\text { - En ambas materias se empezó a manejar el programa } \\
\text { de diseño Google Sketchup para dar formato al boceto. } \\
\text { - Competencias implicadas: Competencia para aprender } \\
\text { a aprender, Tratamiento de la información y }\end{array}$ \\
\hline $\begin{array}{l}\text { 2. Desarrollo en el taller } \\
\text { de Tecnología con } \\
\text { material reciclado y } \\
\text { comprado del robot. } \\
\text { (Ver Fig. 2). }\end{array}$ & Tecnología. & $\begin{array}{l}\text { - El alumnado desarrolló en la materia de Tecnología el } \\
\text { Proyecto de Desarrollo manual del Robot. } \\
\text { - Competencias implicadas: Competencia para aprender } \\
\text { a aprender, Competencia conocimiento y la interacción } \\
\text { con el mundo físico. }\end{array}$ \\
\hline $\begin{array}{l}\text { 3. Desarrollo de un póster } \\
\text { interactivo digital en } \\
\text { lengua inglesa con la } \\
\text { Herramienta Glogster. } \\
\text { (Ver Fig. 3). }\end{array}$ & Lengua inglesa. & $\begin{array}{l}\text { - El alumnado desarrolla un póster explicativo en inglés } \\
\text { con terminología asociada al proyecto. } \\
\text { - Competencias implicadas: Lingüística, Competencia } \\
\text { para aprender a aprender, Tratamiento de la } \\
\text { información y competencia digital. }\end{array}$ \\
\hline 4. Diseño de una & Lengua española. & - El alumnado desarrolla una presentación interactiva \\
\hline
\end{tabular}

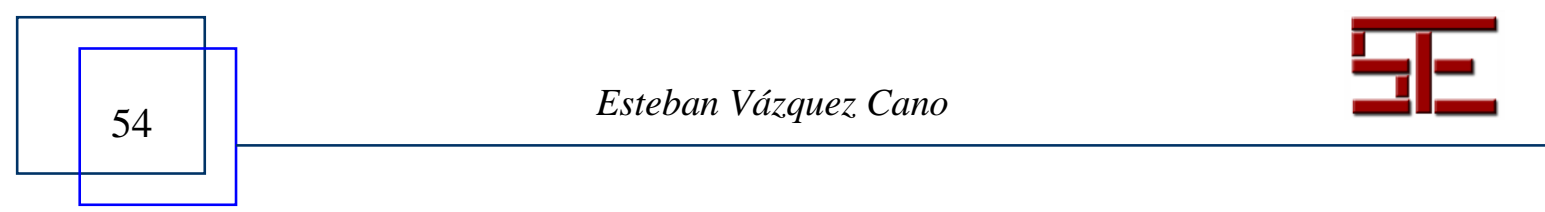




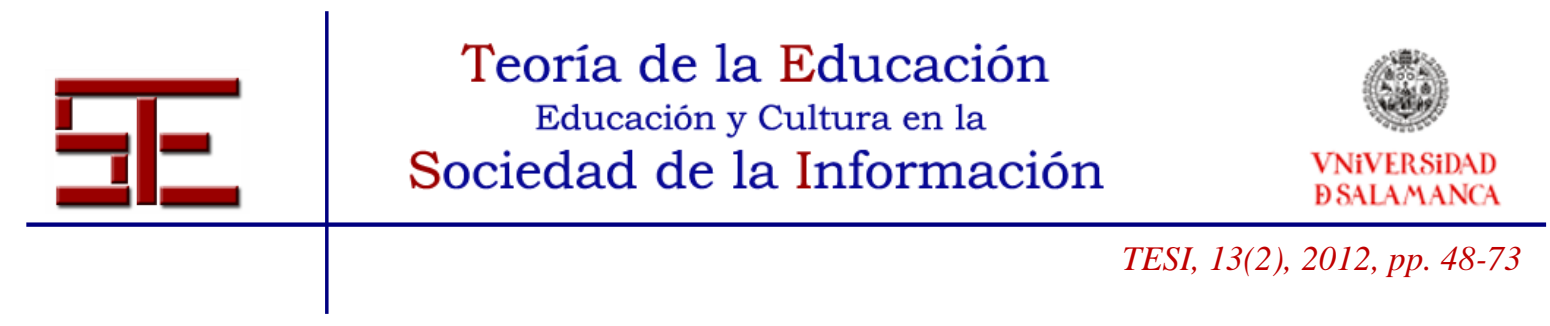

presentación explicativa de todo el proceso con la herramienta Prezi. (Ver Fig. 4).

5. Grabación del proceso de construcción y diseño del robot con la herramienta Windows Movie Maker. (Ver Fig. $5)$. con Prezi en lengua española que explica el desarrollo del proyecto y es explicado en clase de forma oral.

- Competencias implicadas: Lingüística, Competencia para aprender a aprender, Tratamiento de la información y competencia digital.

- El alumnado desarrolla una grabación de las diferentes fases del proyecto con el programa Windows Movie Maker para su posterior uso como videosimulación en otros cursos.

- Competencias implicadas: Competencia para aprender a aprender y Tratamiento de la información y competencia digital.

A continuación, podemos observar las figuras asociadas a cada una de las fases del proyecto:

Figura 1. Fase primera: Alzado del Robot realizado con el programa Google Sketchup

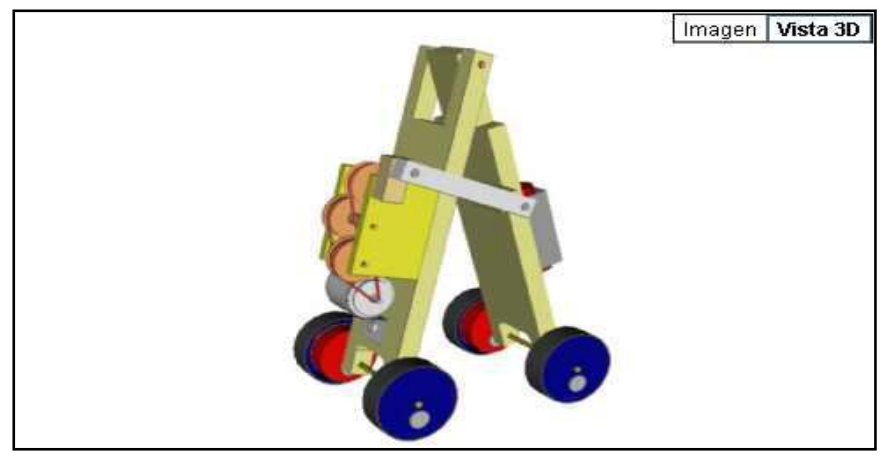

Figura 2. Fase segunda: Robot fabricado con materiales en el taller de Tecnología
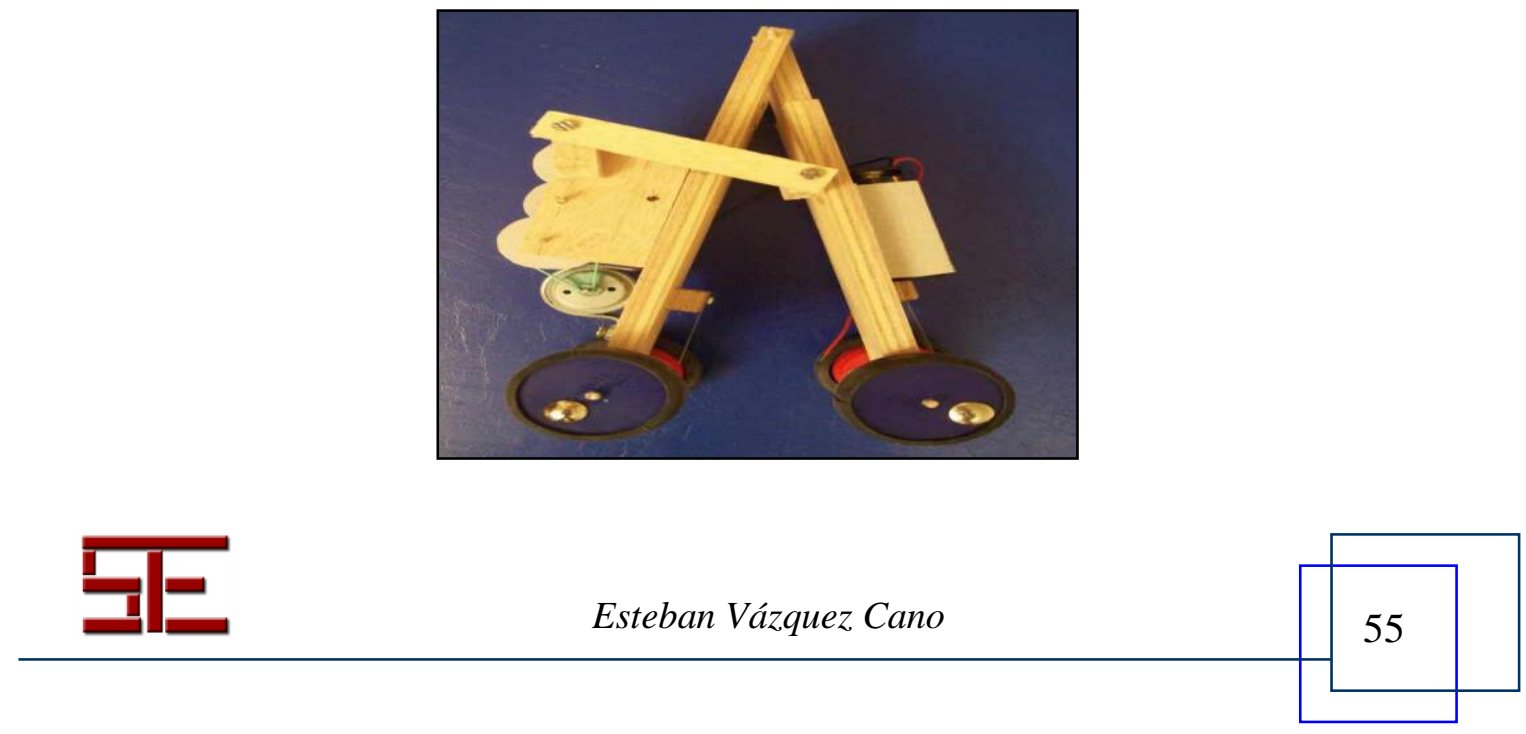


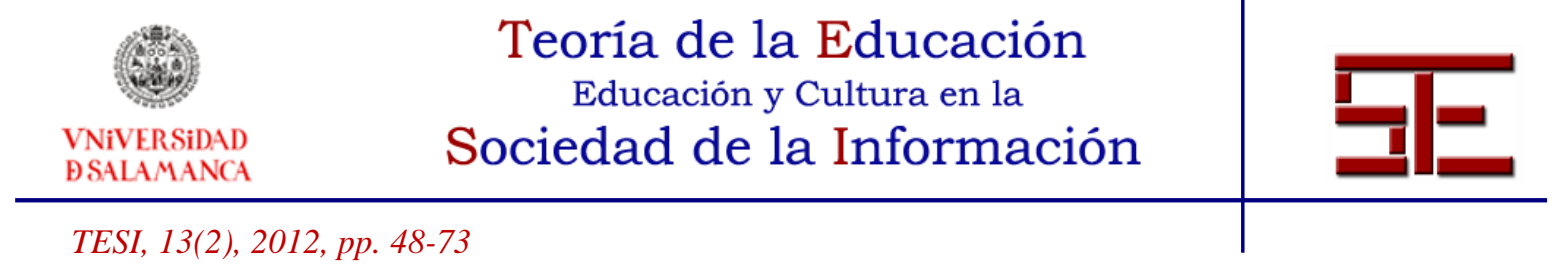

Figura 3. Fase tercera: Presentación del proyecto con Glogster

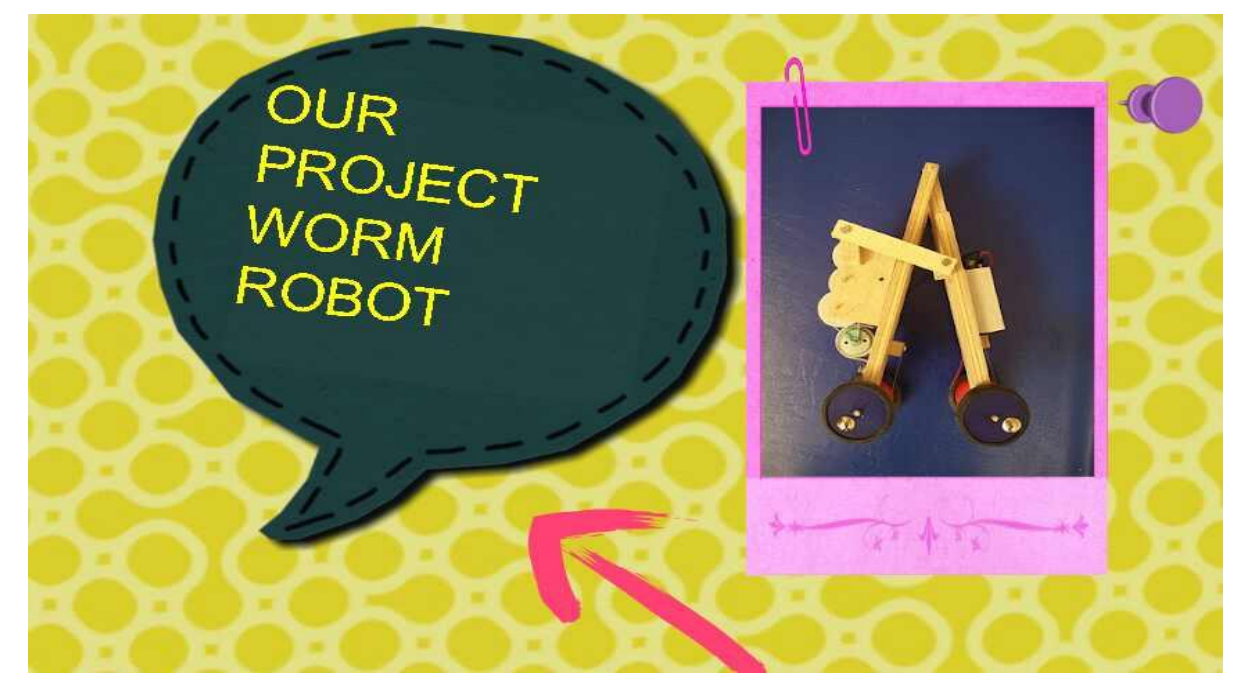

Figura 4. Fase cuarta: Presentación del proyecto con Prezi

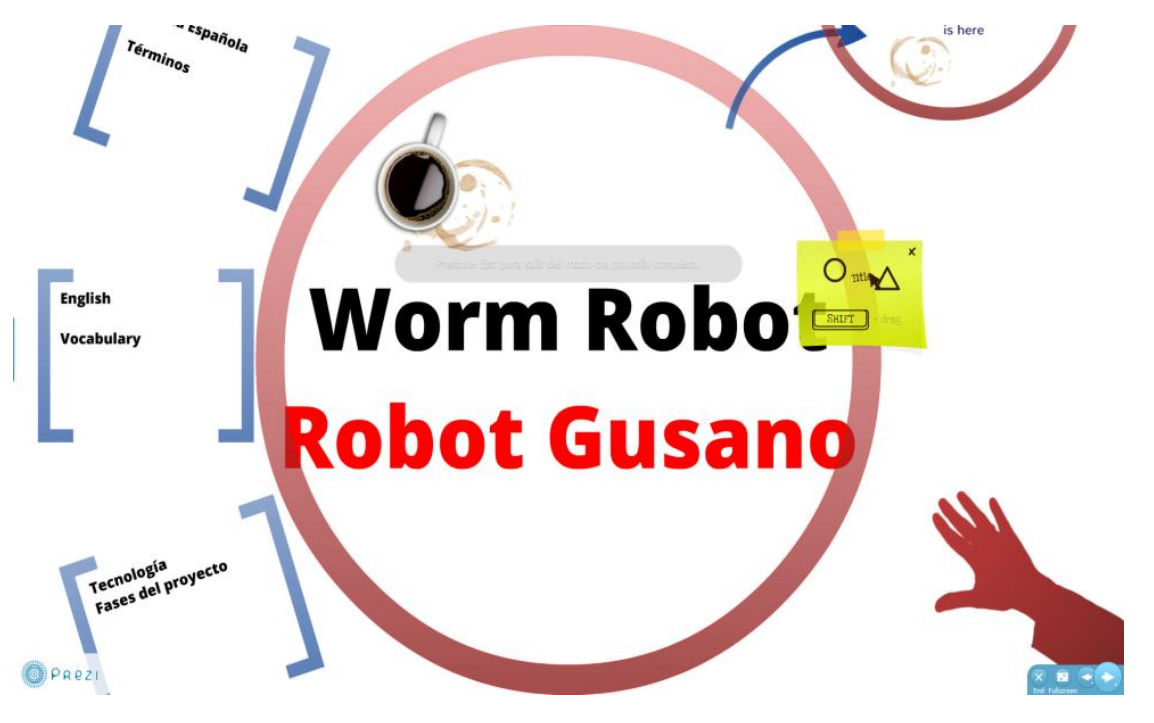




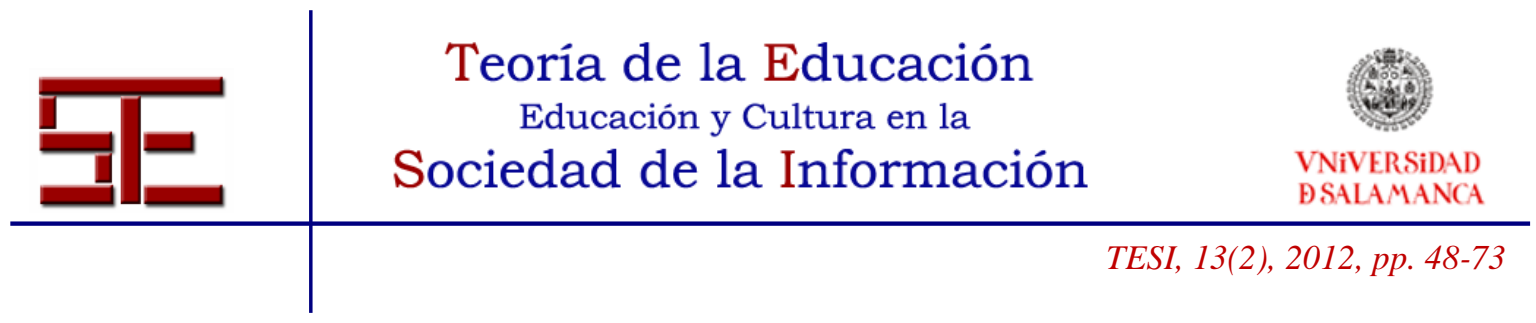

Figura 5. Fase quinta: Presentación del proyecto con Windows Movie Maker

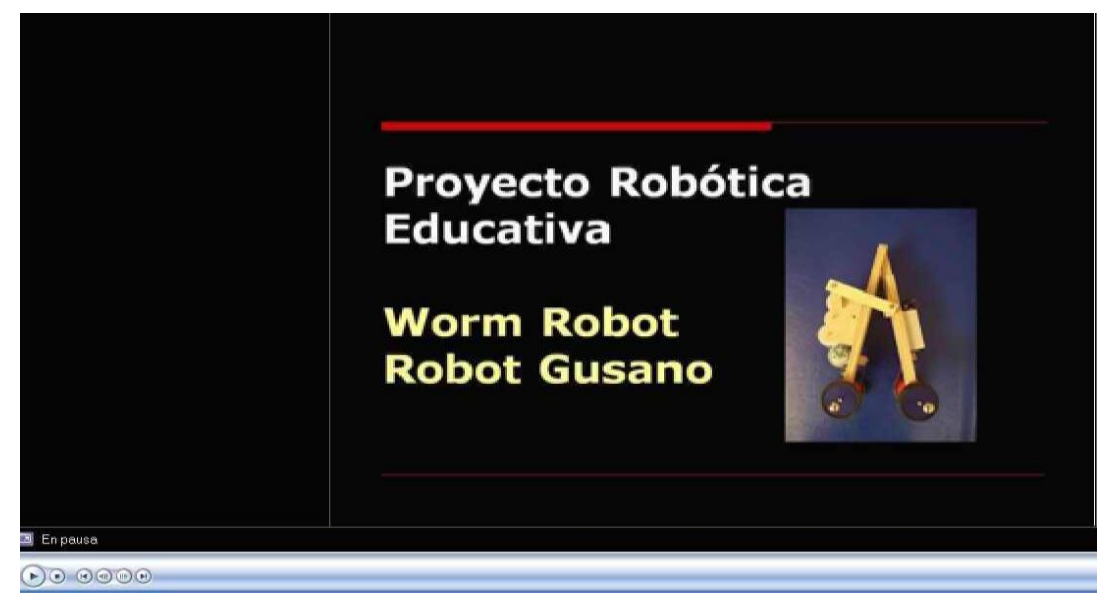

\section{4.- MÉTODO}

El presente trabajo se ha abordado desde un estudio de casos y se encuadra en una metodología de corte cualitativo y cuantitativo. El enfoque cualitativo ha ido encaminado a obtener información relevante sobre la apreciación de los sujetos implicados (profesorado y alumnado) sobre la utilidad y funcionalidad del trabajo por proyectos con base en la robótica educativa. De Pablo, Colas y Villaciervos (2010) mantienen que el uso o integración de las TIC en la educación es complejo y que, por este motivo, las investigaciones sobre esta temática se han ido reorientando hacia estudios de carácter cualitativo, que posibilitan un conocimiento más profundo sobre el impacto que producen. De modo que hemos analizado en profundidad una unidad microcontextual que busca, principalmente, la descripción y comprensión de un grupo reducido de participantes (Bolívar, Domingo \& Fernández, 2001) y sus apreciaciones sobre el uso de la robótica educativa desde la interdisciplinariedad y el apoyo de las herramientas 2.0 en la Enseñanza Secundaria Obligatoria. Con este enfoque de corte cualitativo hemos querido dar respuesta a los dos primeros objetivos de nuestra investigación:

1. Describir la actividad de simulación robótica como parte de un proceso de innovación curricular.

2. Analizar las apreciaciones de los sujetos implicados (profesorado y alumnado) en el desarrollo de la actividad.

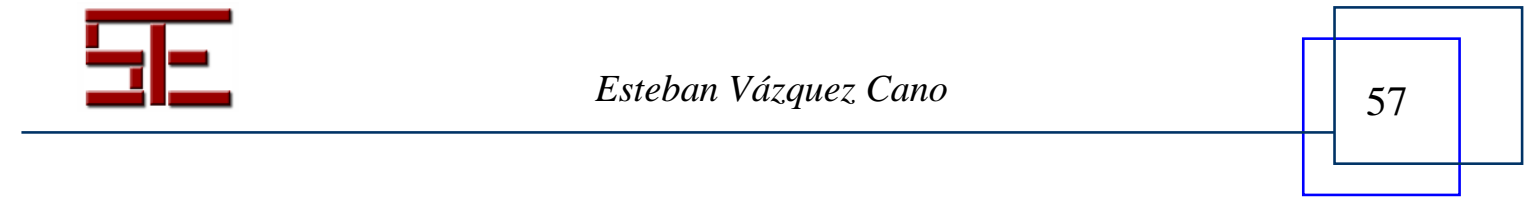




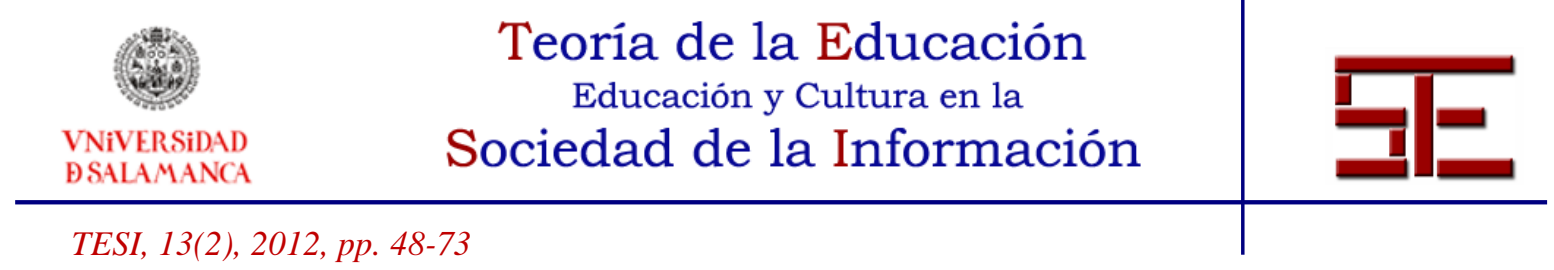

El enfoque cuantitativo se ha abordado desde la comparación de resultados en las pruebas regionales de competencias básicas al alumnado de $2^{\circ}$ curso de la ESO para valorar el grado de mejora con posterioridad al desarrollo del proyecto. Asimismo, se han realizado entrevistas personales a profesorado y alumnado que han servido para valorar la funcionalidad didáctica del programa de robótica educativa y de las herramientas 2.0 en el desarrollo del proyecto y con incidencia especial en el desarrollo de competencias básicas. Con ambos análisis cuantitativos y cualitativos, hemos pretendido dar respuesta a los segundos dos objetivos de la investigación:

3. Comprobar la funcionalidad de las herramientas 2.0 en el desarrollo de proyectos de robótica educativa.

4. Analizar la funcionalidad de los proyectos interdisciplinares con base en la robótica educativa y las TIC en el desarrollo de las competencias básicas.

\section{1.- Participantes y contexto}

La muestra de profesorado ha sido de cuatro docentes pertenecientes a las materias de Taller Tecnológico, Lengua española y Lengua inglesa y Educación Plástica y Visual. El alumnado implicado fue seleccionado de un curso perteneciente a $2^{\circ}$ de la ESO en el que compartían dichas materias y que estaba compuesto por 15 alumnas y 13 alumnos con edades comprendidas entre 13 y 15 años dependiendo de la trayectoria académica del alumnado. El contexto en el que hemos centrado el estudio de casos está situado en la zona norte de Toledo (La Sagra) en un centro educativo con 657 alumnos/as.

\section{2.- Recogida de la información}

La recogida de datos cualitativos se ha realizado mediante visita del investigador al aula con el permiso de la Inspección de Educación para la observación de la actividad. Se utilizaron entrevistas individuales y en grupo al profesorado y al alumnado; lo que nos ha proporcionado una narración extensa de tipo descriptivo y vivencial, en la que afloran los hechos y emociones que han sido más representativos y cruciales para los implicados. Promover la comprensión de los actores principales del proceso formativo es muy importante y supone un medio para mejorar la práctica educativa (Schön, 1992; Hernández \& Vergara, 2004). El alumnado y el profesorado han gozado de libertad para realizar sus narraciones, de forma abierta, crítica y dinámica.

En cuanto a la validez del instrumento utilizado (entrevistas individuales y en grupo), está condicionado por la propia veracidad de la información obtenida (Martínez Miguélez, 2006). Por ello, desde un principio y, a lo largo del proceso, se le fue

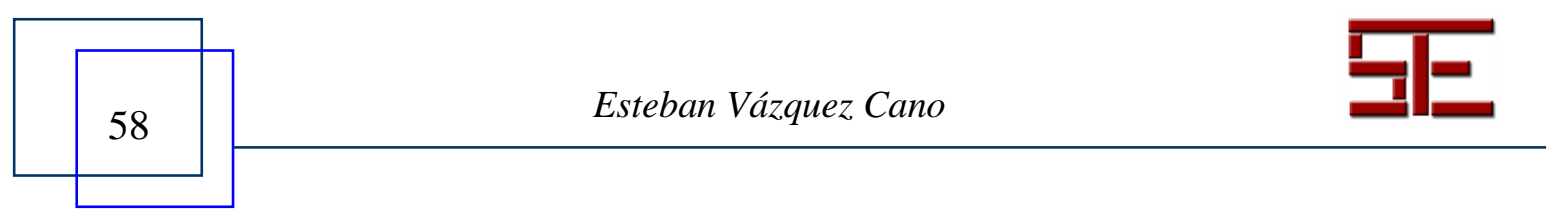




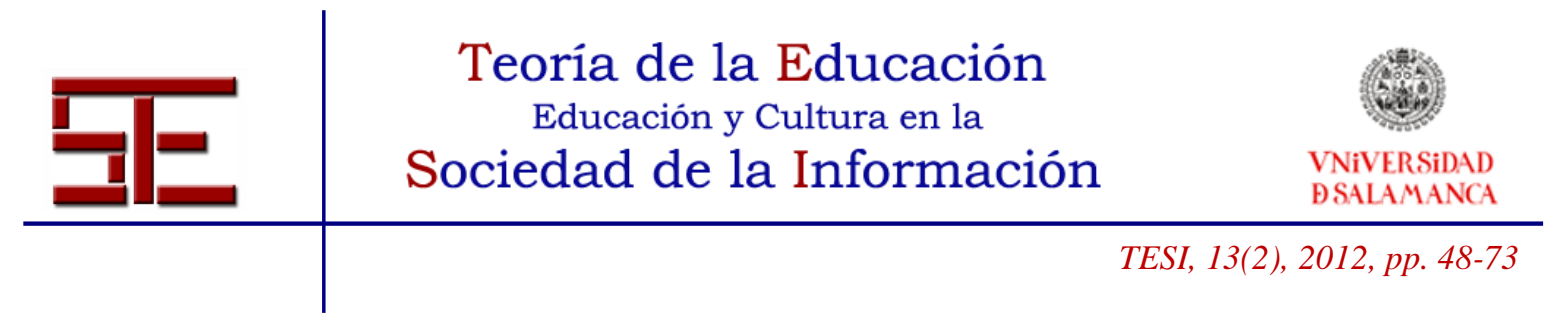

incentivando al alumnado y al profesorado para que desarrollasen con autenticidad las narrativas. De hecho Arandia, Alonso-Olea y Martínez-Domínguez (2010) encuentran que los procesos metodológicos de tipo dialógico contribuyen a que el alumnado tome conciencia de su aprendizaje y propicie la formación de un pensamiento argumentativo y crítico.

Para elaborar los análisis de contenido de la información producida por los estudiantes en las entrevistas, se grabaron y transcribieron las entrevistas al formato .txt, con archivos independientes para cada participante, con el objeto de poder desarrollar su codificación con el programa de "Analysis of Qualitative Data" (AQUAD), versión 5. Finalmente, se ha utilizado el programa "Excel" para concretar el recuento de frecuencias de las subcategorías, de forma individual (para cada participante y código de análisis) y globalmente (atendiendo a las respectivas subcategorías y al conjunto de los participantes). La codificación de los análisis permitió profundizar en el contenido de las narrativas, considerando tanto sus aspectos objetivables como los de carácter subjetivo y emocional (Clandinin \& Connelly, 1994). Los datos globales y desglosados se representan a través de diferentes figuras en el apartado de resultados.

Las entrevistas realizadas al profesorado y alumnado implicado se estructuraron según los ítems de las Tablas 3 y 4.

Tabla 3. Entrevista al profesorado
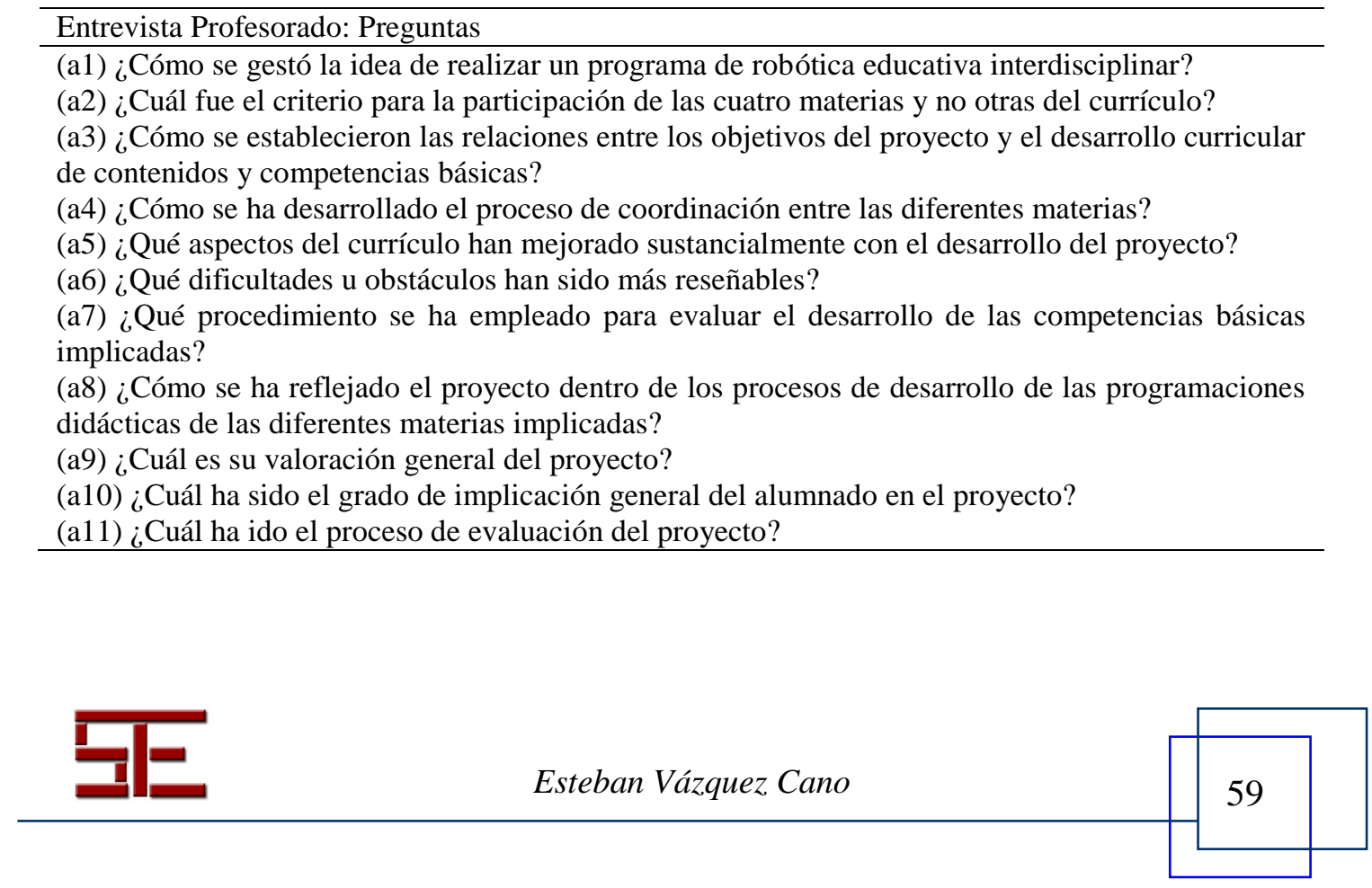


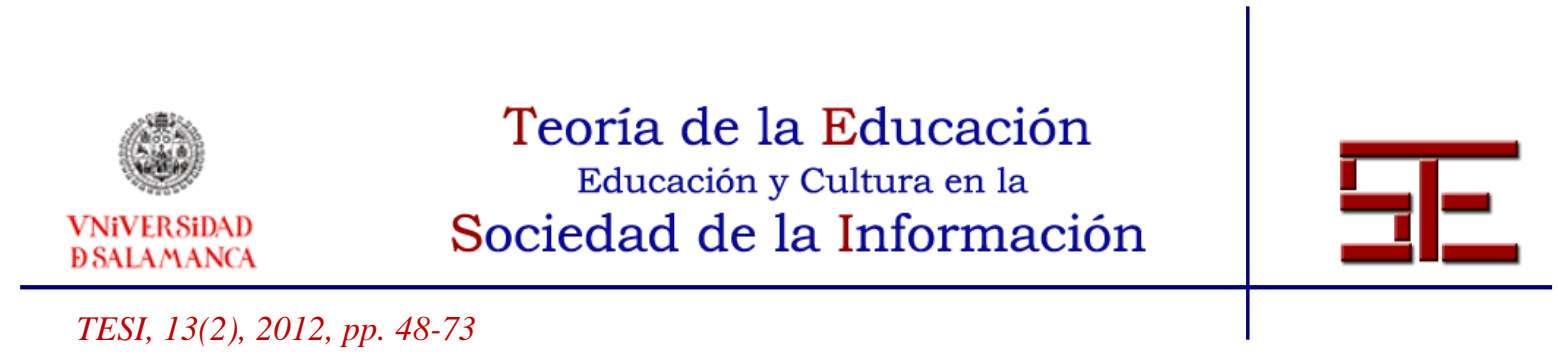

Tabla 4. Entrevista al alumnado

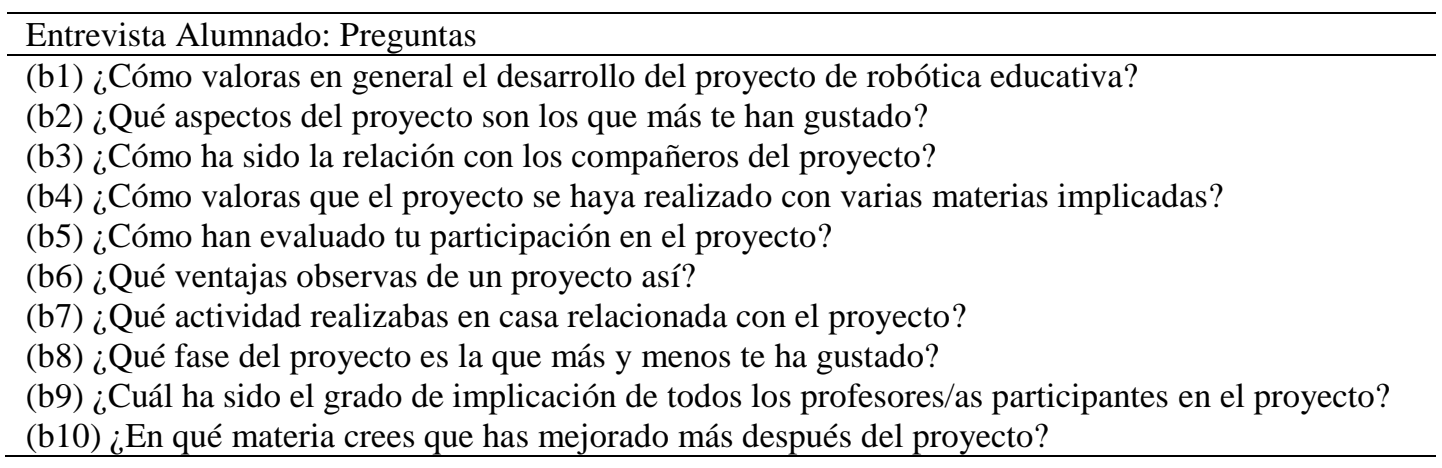

El análisis comparativo de los resultados en competencias básicas se estableció tomando como referencia el sistema de indicadores de competencias realizado por la Oficina de Evaluación de la Junta de Comunidades de Castilla-La Mancha. Para ello, se tomaron los indicadores de los resultados de evaluación de competencias del curso académico 2009/10 con peor resultado y se realizó la comparación con los resultados obtenidos en 2010/11 en esos mismos indicadores y que más relación tenían con el proyecto. A partir de los porcentajes de mejora en aquellos indicadores se validó la funcionalidad de la mejora del proyecto y su incidencia en la adquisición de determinados indicadores de las cuatro competencias básicas con mayor peso en el proyecto de robótica educativa.

\section{5.- RESULTADOS}

En primer lugar, presentamos los resultados cualitativos pertenecientes a las entrevistas a profesorado y alumnado. Con respecto al alumnado, presentamos las subcategorías, señalando el número de frecuencias de los participantes (fp) y el de la cantidad de manifestaciones (fm) que han emitido. Su indicador permite conocer la preponderancia o particularidad que se registró con las respectivas subcategorizaciones. En cuanto a la fiabilidad de los resultados obtenidos, la clave para alcanzarla está asociada a la sistematización lograda en el desarrollo del proceso de investigación y a la consistencia alcanzada en los mismos (Hernández, Fernández \& Baptista, 2006). Presentamos en los siguientes gráficos los hiperónimos descriptivos categorizados según las unidades textuales con mayor frecuencia en relación a las principales apreciaciones del alumnado sobre la utilidad y funcionalidad del proyecto desarrollado.

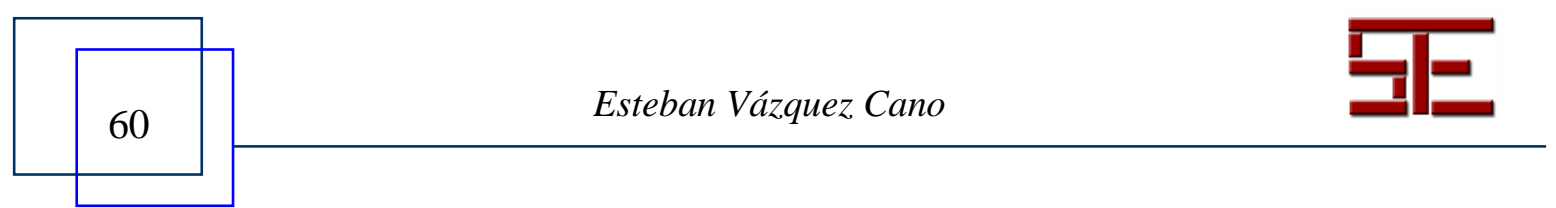




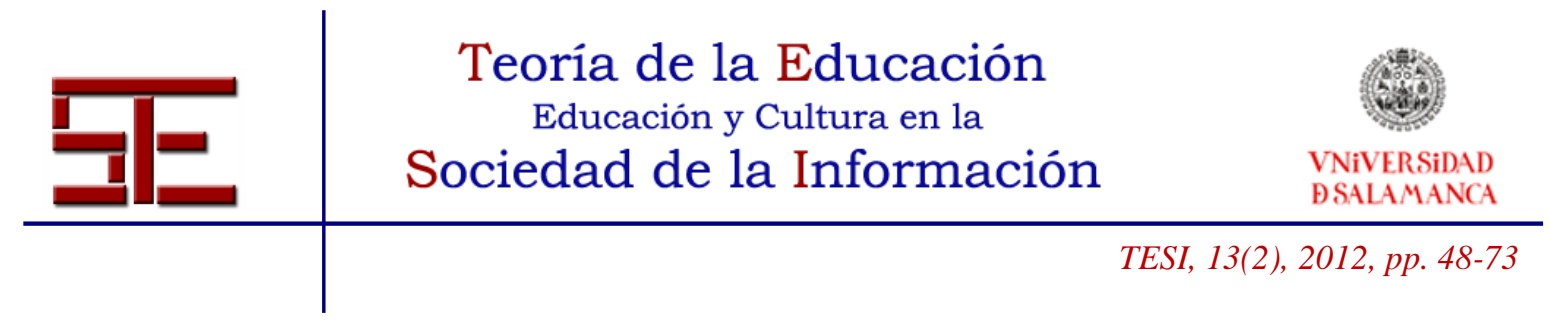

Gráfico 1. Apreciación del alumnado sobre la utilidad del método por proyectos con base en la robótica educativa (Preguntas 1-5)

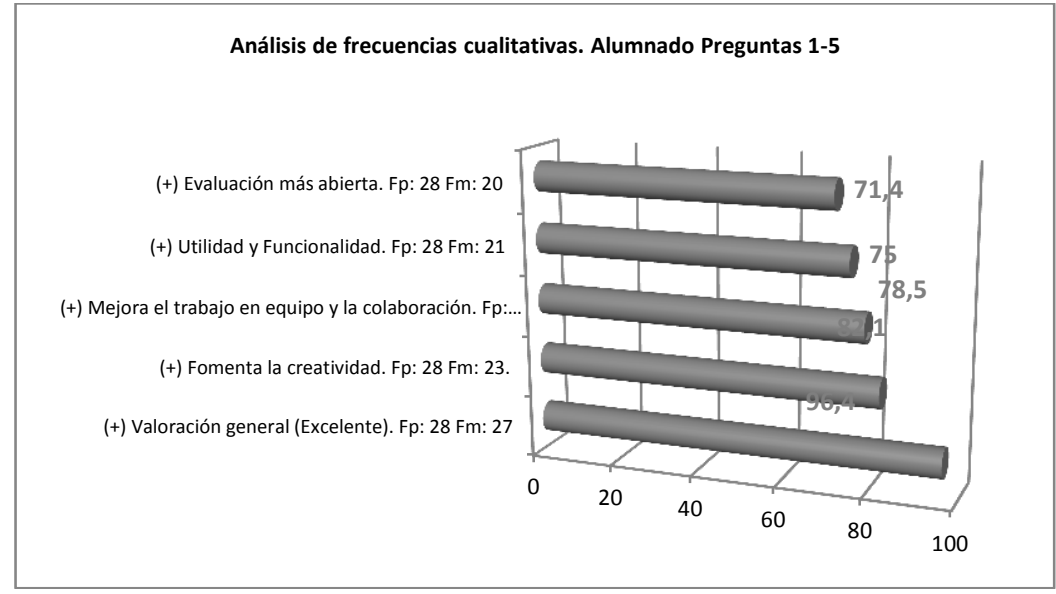

Gráfico 2. Apreciación del alumnado sobre la utilidad del método por proyectos con base en la robótica educativa (Preguntas 6-10)

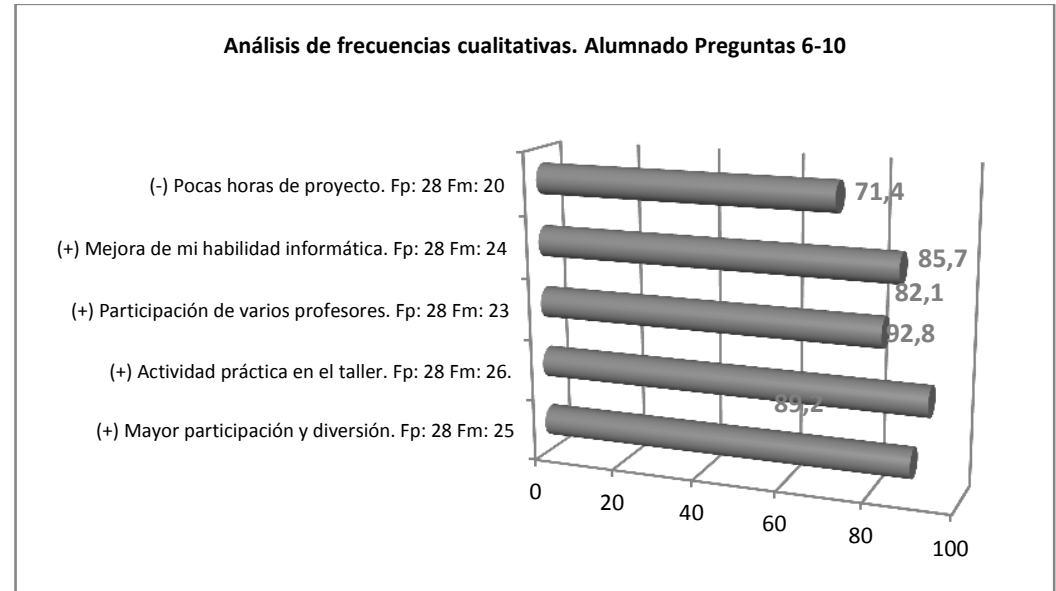

En las entrevistas realizadas al profesorado (Tabla 3) se mostraron opiniones como las siguientes:

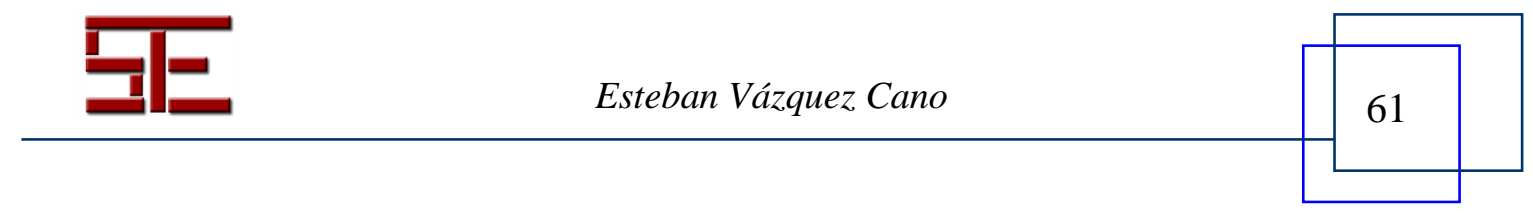




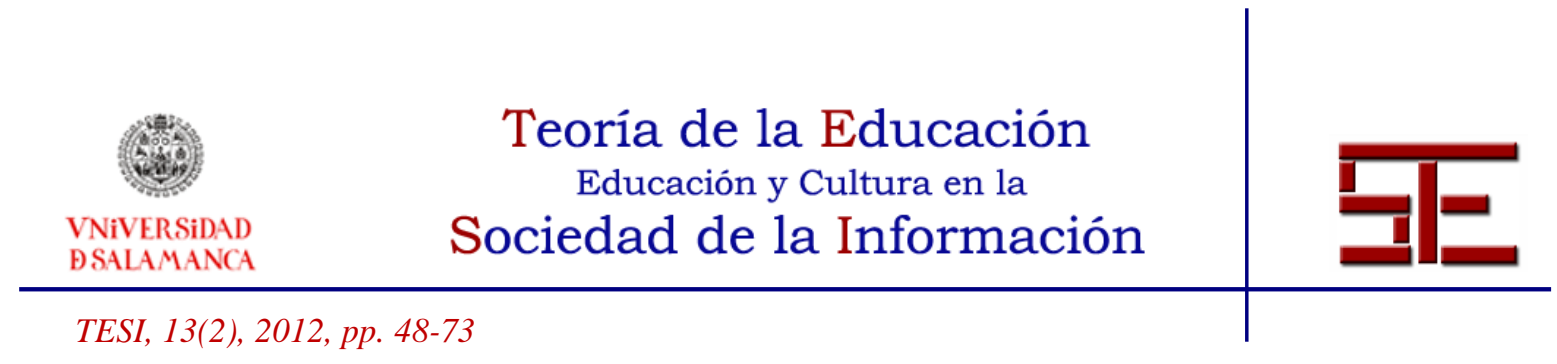

Tabla 5. Resultados entrevista profesorado

\begin{tabular}{|c|c|}
\hline Entrevista & Comentario \\
\hline $\begin{array}{l}\text { (al- Prof. Lengua } \\
\text { española) }\end{array}$ & $\begin{array}{l}\text { "El trabajo por proyectos con base en la robótica educativa se gestó } \\
\text { como propuesta de desarrollar objetivos y competencias básicas de } \\
\text { forma interdisciplinar". }\end{array}$ \\
\hline $\begin{array}{l}\text { (a2- Prof. } \\
\text { Tecnología) }\end{array}$ & $\begin{array}{l}\text { "El criterio para integrar materias del ámbito socio-lingüístico y } \\
\text { científico-tecnológico se adoptó para hacer más significativo e } \\
\text { interdisciplinar los contenidos y destrezas programados". }\end{array}$ \\
\hline (a3-Prof. Inglés) & $\begin{array}{l}\text { "El estudio de las relaciones curriculares entre las diferentes materias } \\
\text { hizo que el profesorado reflexionara sobre los elementos comunes que } \\
\text { comparten diferentes materias y la forma de afrontarlas con una } \\
\text { didáctica común". }\end{array}$ \\
\hline
\end{tabular}

"La coordinación estuvo a cargo de la comisión de coordinación pedagógica del centro educativo en la que se reúnen semanalmente los jefes/as de departamento estableció un cronograma de trabajo para el

(a4-Prof. InglésTecnología)

(a5- Prof. InglésTecnología-LenguaEducación Plástica y Visual)

(a6- Prof. InglésTecnología-LenguaEducación Plástica y Visual)

(a7- Prof. InglésTecnología-LenguaEducación Plástica y Visual) desarrollo de proyectos comunes entre las diferentes materias que persiguieran la consecución de objetivos y competencias básicas de forma transversal. Además, se establecieron reuniones de coordinación en los recreos entre el profesorado implicado para realizar el seguimiento y desarrollo de las actividades".

"Se potencian los contenidos comunes que se enuncian en el artículo 23.7 de la LOE que hacen referencia a la expresión oral y escrita, la comunicación audiovisual y las tecnologías de la Información y la Comunicación y que son referente común para la etapa de Educación Secundaria Obligatoria".

"Los obstáculos y dificultades más reseñados han sido: la falta de horas complementarias para realizar las coordinaciones didácticas entre el profesorado. La falta de suficientes recursos tecnológicos para el desarrollo de las actividades y la falta de cultura de centro para valorar y favorecer el trabajo por proyectos interdisciplinar".

"El procedimiento desarrollado ha consistido en evaluar los indicadores de evaluación de las competencias propuestos por la Oficina de Evaluación de Castilla-La Mancha y que sirven de referencia para los exámenes de adquisición de competencias realizados por la comunidad autónoma".

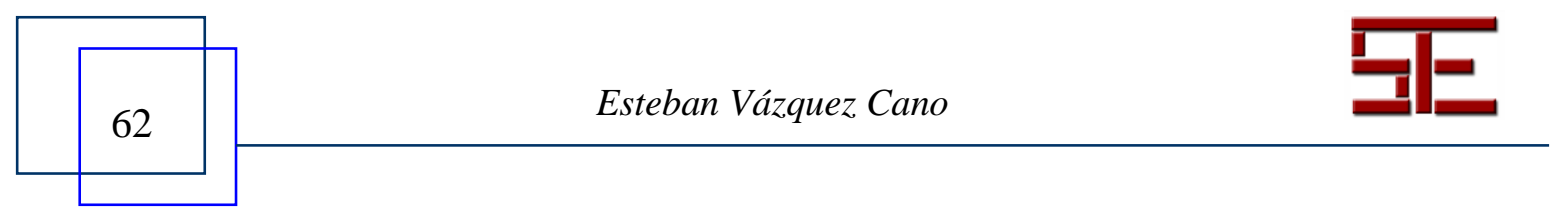




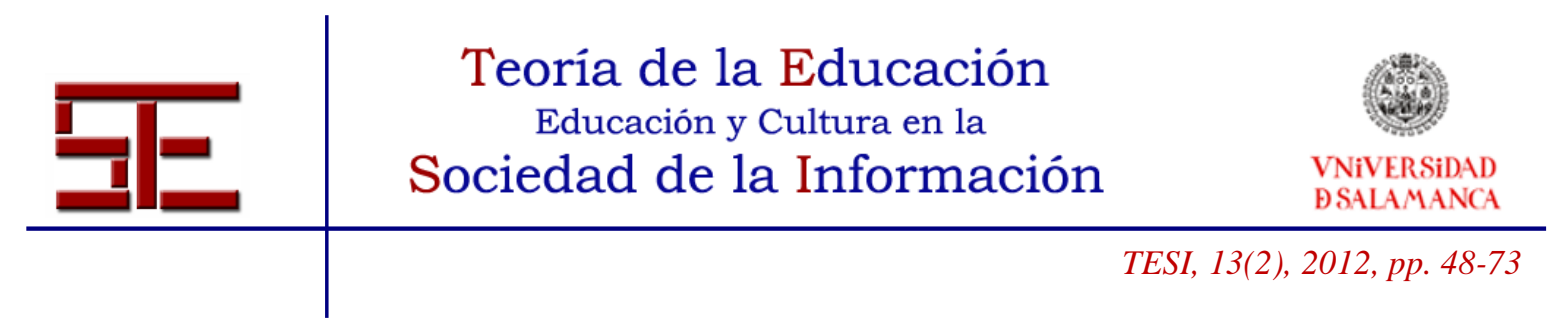

(a8- Prof. InglésTecnología-LenguaEducación Plástica y Visual)

(a9- Prof. InglésTecnología-LenguaEducación Plástica y Visual)

(a10- Prof. InglésTecnología-LenguaEducación Plástica y Visual)

(a11- Prof. InglésTecnología-LenguaEducación Plástica y Visual)
"El procedimiento de reflejar el proyecto en las programaciones didácticas de las diferentes materias se realizó durante la primera evaluación mediante inclusión por acta de departamento didáctico de los objetivos generales del proyecto, su cronograma de trabajo y el procedimiento de evaluación".

"Los cuatro profesores entrevistados consideran el desarrollo del proyecto como muy positivo y enriquecedor tanto para el alumnado como para el profesorado. Los aspectos que más han resaltado han sido: a) El enriquecimiento profesional que ha supuesto trabajar de forma conjunta. b) La mejora de la metodología asociada a cada materia. c) El fomento del trabajo en grupo. d) Colaboración haciendo efectivo el artículo 91 de la LOE".

"El profesorado de las cuatro materias considera que el alumnado ha estado muy motivado e implicado en el proyecto".

"El proceso de evaluación del proyecto se ha realizado mediante memoria descriptiva de objetivos y competencias tratados y se ha reflejado en cada una de las memorias de cada departamento didáctico. La nota obtenida por el alumnado ha sido reflejada ponderadamente en las notas de las tres evaluaciones".

Tomando como referencia el sistema de indicadores de la Junta de Comunidades de Castilla-La Mancha para el desarrollo de las competencias básicas (Oficina de Evaluación), se estableció un patrón de comparación entre los resultados de los alumnos en $2^{\circ}$ curso entre los cursos académicos 2009/10 y 2010/11. Para ello, se recurrió al análisis de los indicadores evaluados en las cuatro competencias implicadas y se obtuvieron los resultados del informe específico del centro (Figura 6).

Figura 6. Extracto del informe de competencias analizado (Oficina de Evaluación)
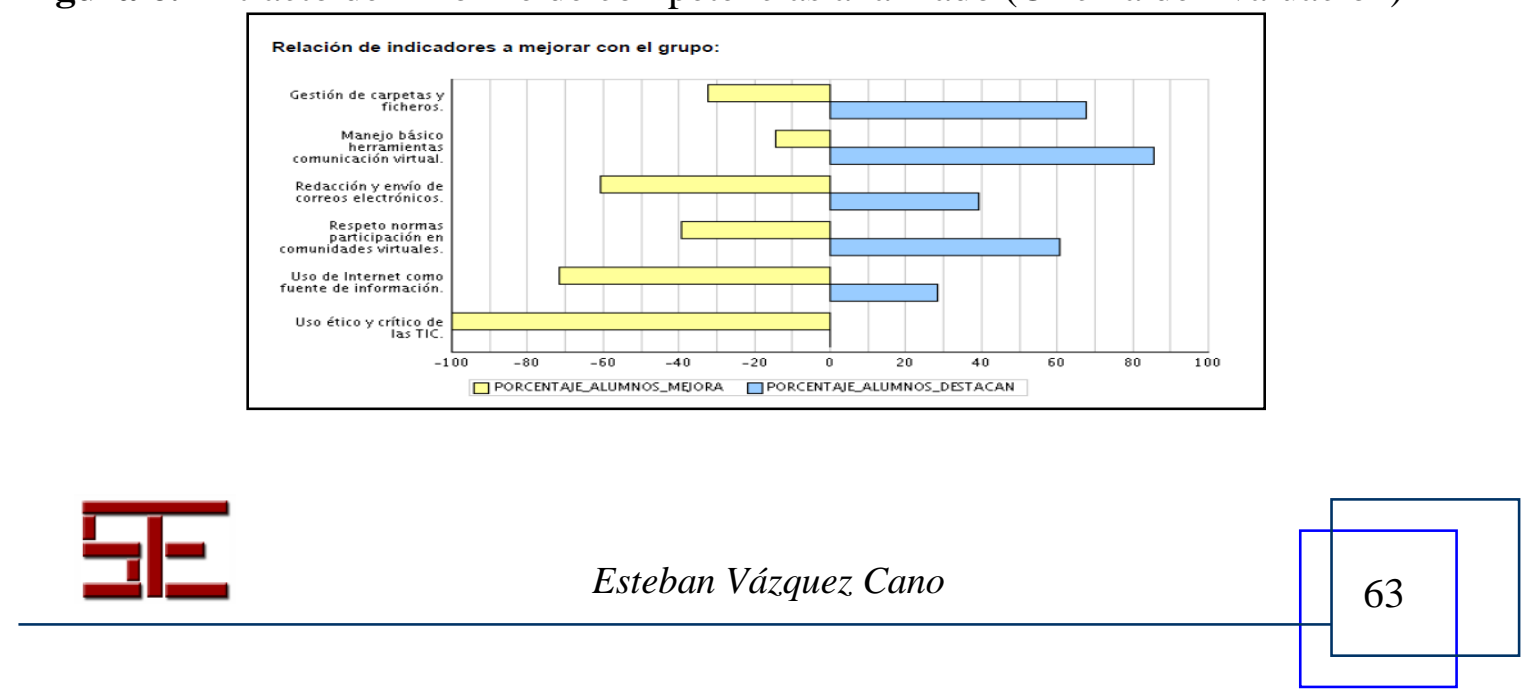


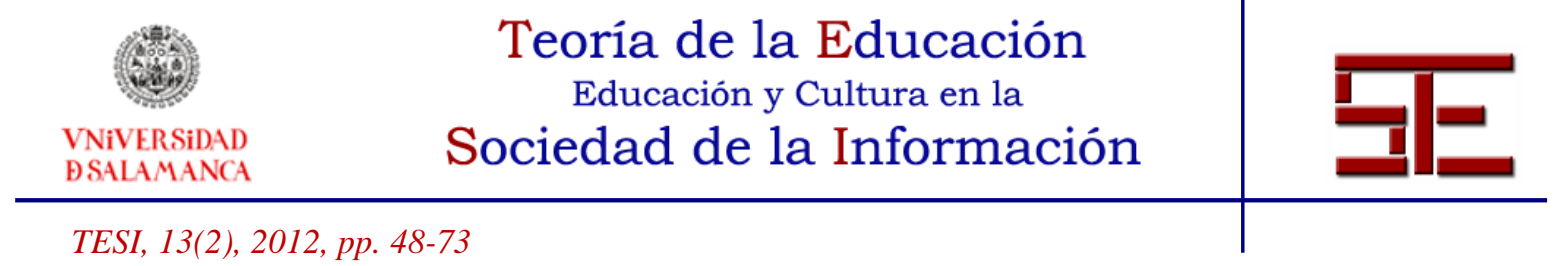

Mediante este informe se identificaron los indicadores en los que el alumnado había obtenido peores resultados y se cotejaron con las pruebas realizadas a los mismos alumnos/as una vez desarrollado el proyecto con base en la robótica educativa. Se establecieron los porcentajes de mejora del alumnado con respecto a las pruebas iniciales.

En la tabla que mostramos, a continuación, presentamos los cuatro indicadores de las cuatro competencias implicadas que mayor porcentaje de mejora han obtenido.

Tabla 6. Porcentaje de mejora por indicadores de competencias básicas

\begin{tabular}{|c|c|c|c|c|}
\hline \multirow{2}{*}{$\begin{array}{c}\text { COMPETENCIAS } \\
\text { Lingüística }\end{array}$} & \multicolumn{4}{|c|}{$\begin{array}{c}\text { INDICADORES } \\
\text { (mostramos los cuatro indicadores con mayor porcentaje de mejora) } \\
\text { Fuente. Oficina de Evaluación. JCCM }\end{array}$} \\
\hline & $\begin{array}{l}\text { Identificación de } \\
\text { las ideas } \\
\text { principales y } \\
\text { secundarias. }\end{array}$ & $\begin{array}{l}\text { Establecimiento de } \\
\text { relaciones causales. }\end{array}$ & $\begin{array}{l}\text { Presentación } \\
\text { multimedia de un } \\
\text { contenido. }\end{array}$ & $\begin{array}{l}\text { Uso del } \\
\text { vocabulario } \\
\text { específico. }\end{array}$ \\
\hline$\%$ mejora & $+23 \%$ & $+12 \%$ & $+22 \%$ & $+24 \%$ \\
\hline $\begin{array}{l}\text { Aprender a } \\
\text { Aprender }\end{array}$ & $\begin{array}{l}\text { Identificación de } \\
\text { obstáculos e } \\
\text { interferencias. }\end{array}$ & $\begin{array}{l}\text { Formulación y } \\
\text { resolución de } \\
\text { problemas. }\end{array}$ & $\begin{array}{l}\text { Uso de Internet } \\
\text { como fuente de } \\
\text { información. }\end{array}$ & $\begin{array}{l}\text { Autoevaluación del } \\
\text { proceso y el } \\
\text { resultado. }\end{array}$ \\
\hline$\%$ mejora & $+13 \%$ & $+31 \%$ & $+28 \%$ & $+18 \%$ \\
\hline $\begin{array}{l}\text { Tratamiento de la } \\
\text { información y } \\
\text { competencia digital }\end{array}$ & $\begin{array}{l}\text { Trabajo con } \\
\text { ficheros en red. }\end{array}$ & $\begin{array}{l}\text { Elaboración de un } \\
\text { guión previo a la } \\
\text { presentación. }\end{array}$ & $\begin{array}{l}\text { Representación de } \\
\text { dibujos y edición de } \\
\text { imágenes. }\end{array}$ & $\begin{array}{c}\text { Presentación } \\
\text { multimedia de un } \\
\text { contenido. }\end{array}$ \\
\hline \% mejora & $+25 \%$ & $+21 \%$ & $+22 \%$ & $+14 \%$ \\
\hline $\begin{array}{l}\text { Competencia en el } \\
\text { conocimiento y la } \\
\text { interacción con el } \\
\text { mundo físico }\end{array}$ & $\begin{array}{l}\text { Organización en } \\
\text { un mapa } \\
\text { conceptual, } \\
\text { esquema. }\end{array}$ & $\begin{array}{l}\text { Distribución de un } \\
\text { proceso en fases, } \\
\text { tareas y } \\
\text { responsables. }\end{array}$ & $\begin{array}{c}\text { Uso de la } \\
\text { observación y } \\
\text { experimentación. }\end{array}$ & $\begin{array}{l}\text { Análisis de causas, } \\
\text { interrelaciones y } \\
\text { riesgos. }\end{array}$ \\
\hline$\%$ mejora & $+31 \%$ & $+13 \%$ & $+18 \%$ & $+19 \%$ \\
\hline
\end{tabular}

\section{6.- ANÁLISIS E INTERPRETACIÓN DE LOS RESULTADOS}

Los resultados obtenidos se han organizado en dos grandes bloques: apreciación de alumnado y profesorado sobre la funcionalidad, utilidad y repercusión del proyecto y repercusión de la metodología de enseñanza-aprendizaje en la mejora del desarrollo de las competencias básicas. Con respecto al primer bloque, podemos establecer unas consideraciones bastantes positivas.

El alumnado en una gran mayoría (Fp:28 Fm:27) considera que el proyecto ha supuesto una mejora del trabajo en equipo, de la colaboración y del fomento de la creatividad

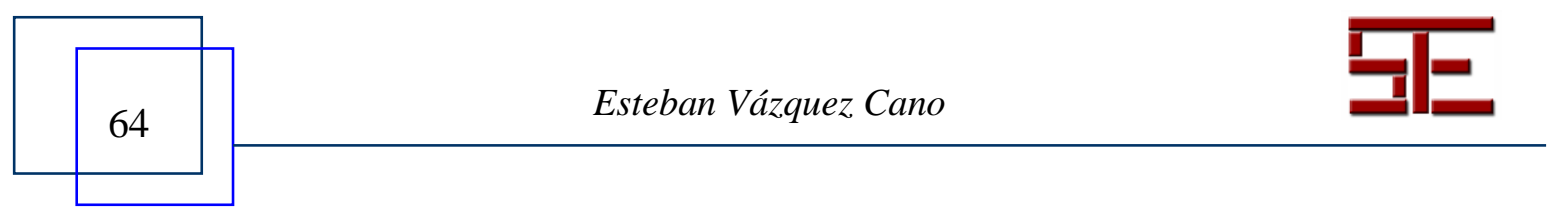




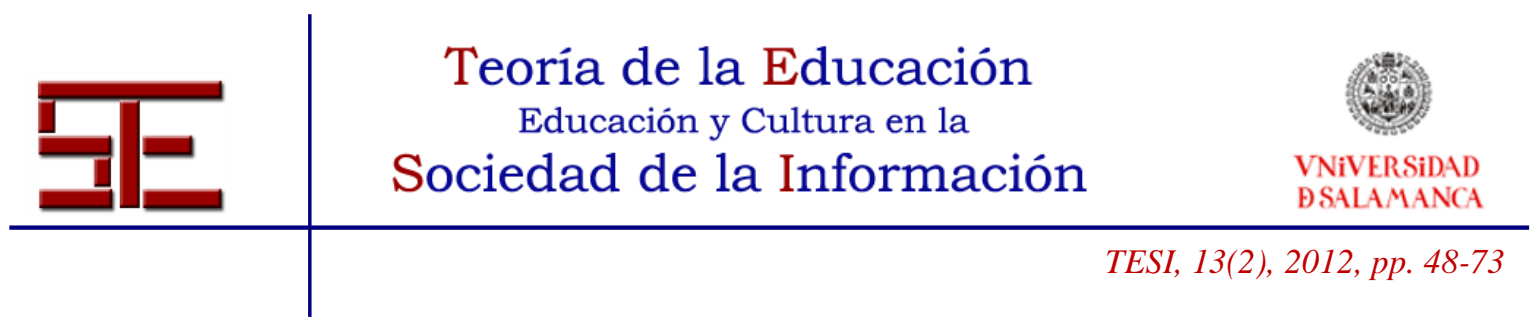

(Fp:28 Fm:22-23). Estas actividades insertadas en proyectos que abarcan contenidos y objetivos de diferentes materias resultan muy productivas tanto a nivel curricular como de clima de aula y personal del alumnado. Los propios alumnos resaltan que después de su desarrollo han visto mejoradas habilidades informáticas y linguiísticas (Fp:28 Fm:24). Se ven motivados por la participación en actividades prácticas en el aula-taller y la posibilidad de realizar actividades interrelacionadas entre materias diferentes (Fp:28 Fm:28-23).

La robótica educativa es una actividad muy atrayente para los alumnos, ya que conlleva una manipulación práctica de recursos y materiales, si además se conjuga con el uso de las TIC de forma operativa y lingüística; la implicación del alumnado se hace mayor y su esfuerzo se aplica a diferentes materias que tienen implicaciones directas en los procesos tecnológicos y mecánicos que confluyen en la robótica educativa e industrial. Los alumnos se ven tan motivados que reseñan como principal dificultad u obstáculo del proyecto la falta de horas para su desarrollo y su deseo de que se extendiera en el tiempo (Fp:28 Fm:20). Los alumnos perciben muy positivamente que sean varios profesores y materias los que participen en el proyecto ya que creen que así se asimilan mejor contenidos que son comunes a las diferentes materias y, que al ser evaluados conjuntamente, mejoran su rendimiento y resultados (Fp:28 Fm:23).

Con respecto a los resultados de las entrevistas del profesorado, cabe reseñar que los cuatro profesores manifiestan que el proyecto con base en la robótica educativa ha servido de eje vertebrador de los contenidos transversales enunciados en el artículo 23.7 de la LOE (Art. 23-7: "Sin perjuicio de su tratamiento específico en algunas de las materias de la etapa, la comprensión lectora, la expresión oral y escrita, la comunicación audiovisual, las tecnologías de la información y la comunicación y la educación en valores se trabajarán en todas las áreas"). Estos contenidos transversales son difíciles de conseguir de forma aislada por cada materia y su trabajo interdisciplinar con este proyecto ha supuesto una mejoría sustancial (a5).

La implicación del centro y su equipo directivo se ha apuntado como una de las condiciones esenciales para que este tipo de proyectos puedan tener éxito. Se precisa que el equipo directivo fomente reuniones de coordinación en horario escolar y que se disponga de espacios comunes para el desarrollo de las actividades en la que se pueda colaborar entre, al menos, dos profesores de diferentes materias (a4).

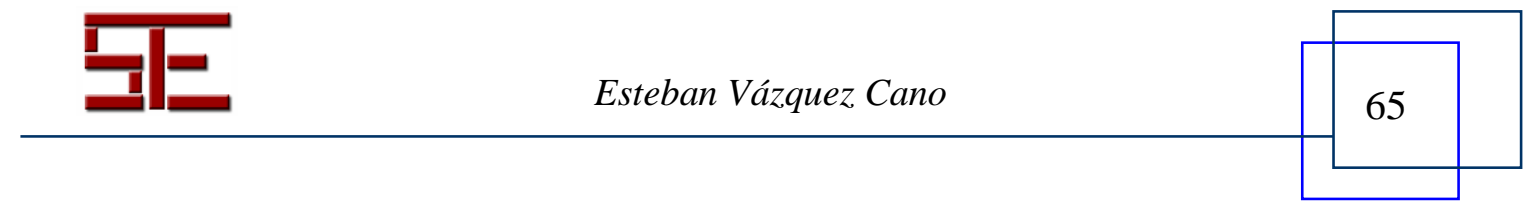




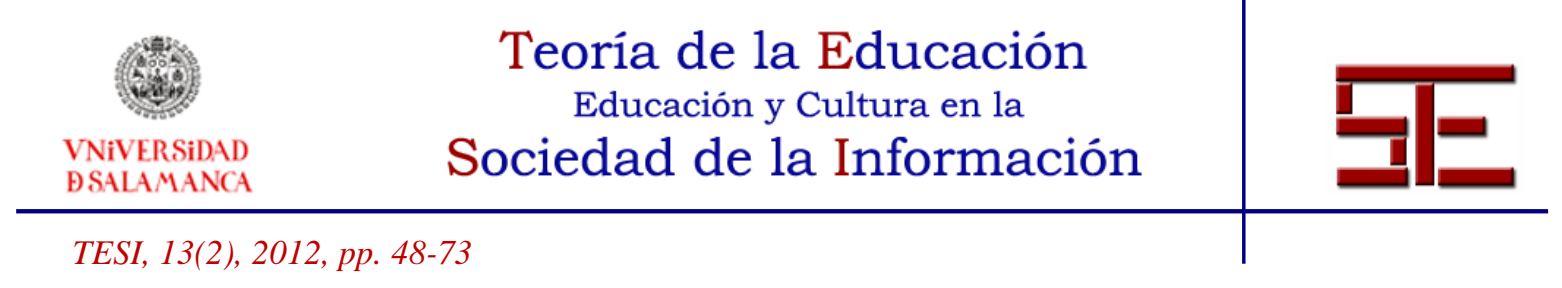

Se ha apuntado como una de las mayores contribuciones del proyecto, el desarrollo conjunto de las competencias básicas. Éste es uno de los aspectos que más controversia y reticencias suscita en el profesorado. Todavía no se han instaurado unas prácticas claras para el desarrollo conjunto e interdisciplinar de las competencias básicas. El profesorado ha valorado muy positivamente que con este tipo de proyectos se promueve un verdadero trabajo interdisciplinar y se puedan desarrollar competencias y objetivos de etapa de forma conjunta y con una mayor incidencia en las destrezas y habilidades del alumnado (a7).

Asimismo, el profesorado ha apuntado que con este tipo de metodologías se desarrolla un adecuado clima de clase y de trabajo conjunto tal y como especifica el artículo 91 de la LOE donde se establece en el punto 91.2 lo siguiente: "Los profesores realizarán las funciones docentes bajo el principio de colaboración y trabajo en equipo" (a9).

La principal dificultad u obstáculo para el profesorado es la falta de horas de coordinación y la falta espacios adecuados para desarrollar el proyecto con programas y equipos informáticos adecuados (a6).

Los resultados con respecto a la mejora de los indicadores de las cuatro competencias básicas implicadas han sido sustanciales y, en comparación con las pruebas del curso académico anterior, se han visto claramente mejorados al menos cuatro indicadores que anteriormente habían obtenido resultados poco aceptables. Los indicadores que más han mejorado se pueden agrupar en tres ámbitos: Lingüístico, para lo que más han portado los programas Prezi y Glogster. Organización del trabajo, para lo que ha aportado mucho la organización en fases del proyecto de forma interdisciplinar entre las diferentes materias implicadas. Tratamiento de la información y del diseño digital, para lo que fue clave el manejo del programa Google Sketchup y las presentaciones en diferentes lenguas del desarrollo y terminología específica del proyecto.

A continuación, mostramos los resultados de los indicadores de las cuatro competencias básicas implicadas en el proyecto agrupando el porcentaje de mejora de los indicadores en los tres ámbitos anteriormente comentados:

-Tratamiento de la información y diseño digital.

-Ámbito lingüístico.

-Organización del trabajo.

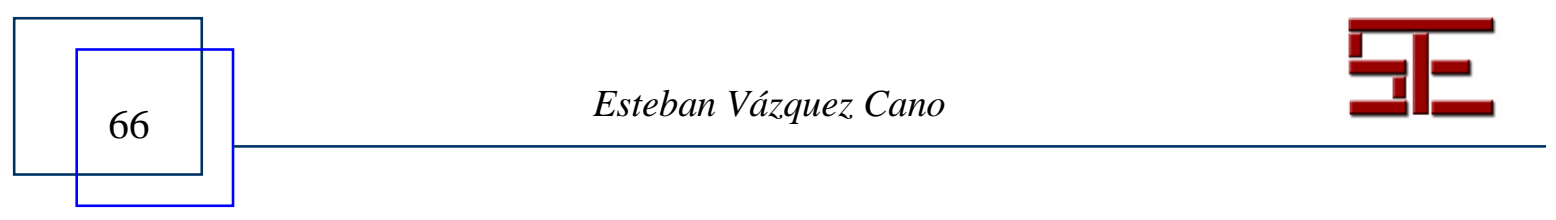




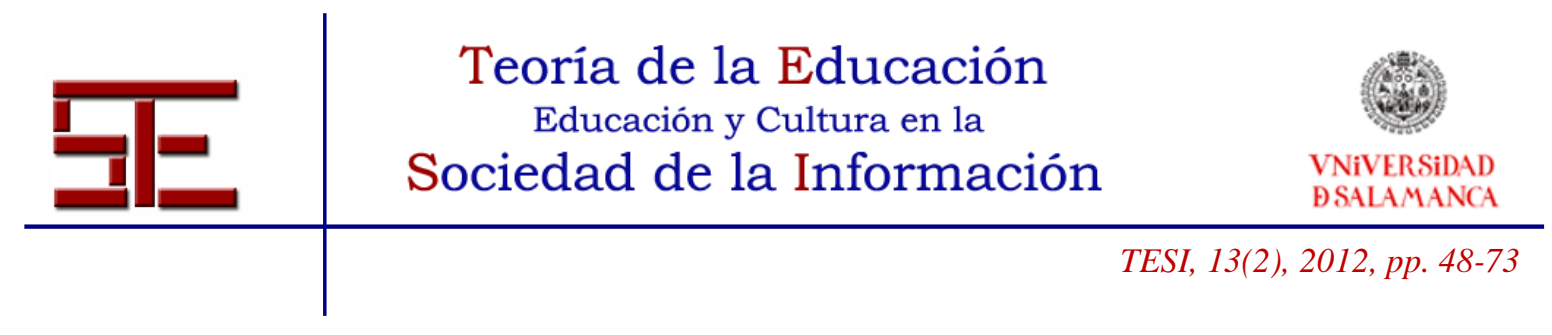

Gráfico 3. Porcentaje agrupado de indicadores de competencias mejorados con el proyecto

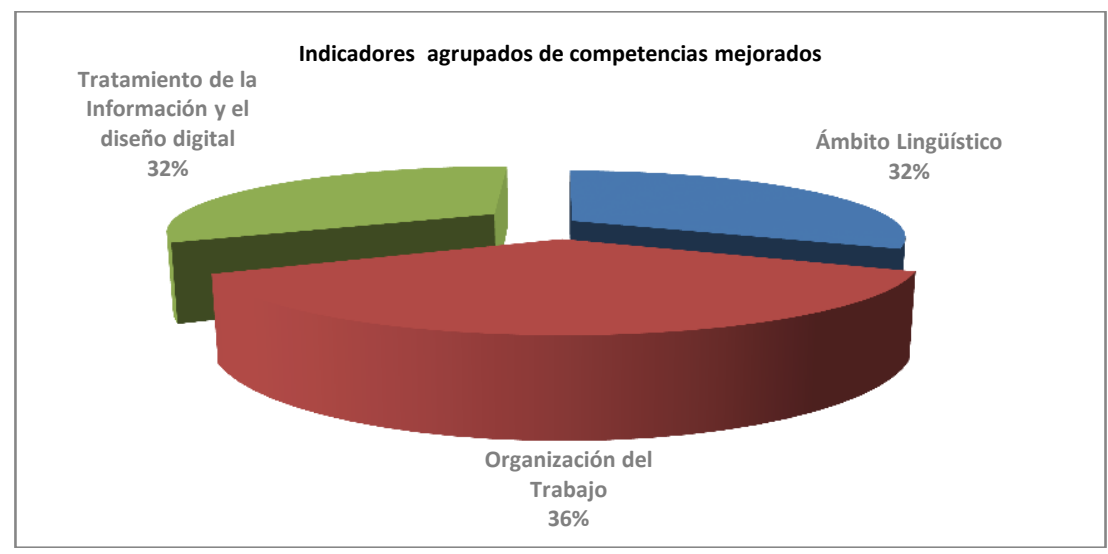

\section{7.- CONCLUSIONES}

La experiencia que aquí se describe y analiza con base en la robótica educativa y el apoyo en su desarrollo con herramientas 2.0 de forma interdisciplinar es una innovación curricular y didáctica que arroja unos resultados muy positivos en el desarrollo de contenidos y en el afianzamiento de indicadores claves en el desarrollo de cuatro competencias básicas: lingüística, aprender a aprender, tratamiento de la información y competencia digital y conocimiento y la interacción con el mundo físico. Hemos podido comprobar cómo la combinación en un mismo proyecto de principios de la robótica educativa junto con herramientas 2.0 y el trabajo interdisciplinar entre materias ha promovido ambientes de aprendizaje competenciales y funcionales donde los estudiantes han adquirido habilidades para estructurar investigaciones y resolver problemas concretos, aplicando conceptos teóricos de forma práctica para dar respuesta eficiente a los entornos cambiantes del mundo actual.

Los logros principales que hemos podido constatar se han centrado fundamentalmente en la resolución de problemas y en la aplicación del método científico para probar y generar nuevas hipótesis sobre posibles soluciones. Asimismo, al ser el proceso interdisciplinar e implicar a cuatro materias, dos de ellas del ámbito lingüístico, el alumnado ha mejorado indicadores clave de la competencia lingüística (española e

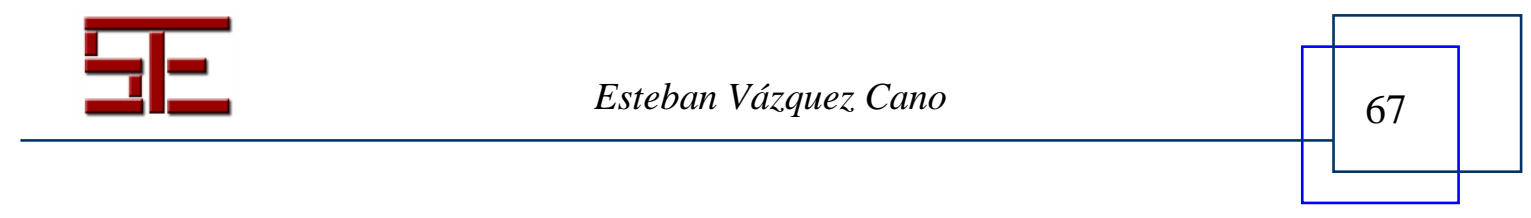




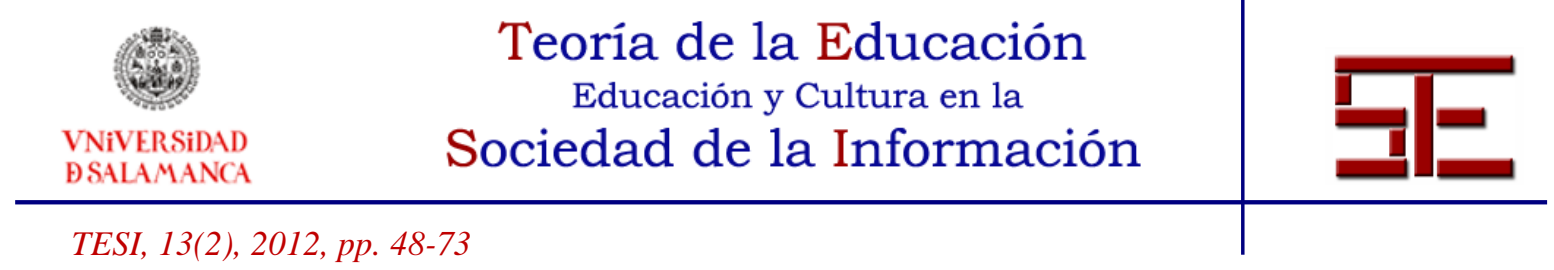

inglesa) al utilizar vocabulario especializado y construir sus propias concepciones acerca del significado de cada objeto manipulado. Además, las presentaciones interactivas con la realización de un póster digital en inglés con la herramienta digital Glogster y la explicación del proyecto en lengua española con la herramienta de realización de presentaciones interactivas Prezi han supuesto la mejora sustancial de indicadores de las cuatro competencias básicas.

El alumnado se ha visto impelido a compartir sus producciones con el resto de compañeros y de forma interdisciplinar entre las diferentes materias implicadas; lo que supone el desarrollo del sentido crítico acerca de sus propias creaciones y las de sus compañeros, produciéndose un intercambio valioso de experiencias que contribuyen al aprendizaje por medio del análisis y la crítica constructiva. El proyecto también ha contribuido a interiorizar diversos conceptos tecnológicos, tales como: diseño y construcción de prototipos propios o modelos que simulan objetos ya creados por el hombre, aplicación de sensores, estrategias de programación, control y sincronización de procesos. Estas dinámicas se han desarrollo mayoritariamente en un ambiente lúdico que ha permitido el desarrollo de la autoestima y las relaciones interpersonales. Finalmente el trabajo en pequeños grupos ha favorecido la creación de una conciencia colectiva y cooperativa que valora el trabajo de los compañeros y reconoce la interdependencia que tienen unos de otros para concluir el proyecto con éxito.

\section{8.- LIMITACIONES Y PROSPECTIVA}

Este estudio se ha limitado a un estudio de casos que describe un proyecto de robótica educativa en un grupo de alumnos/as de la ESO. En futuras investigaciones se debería afrontar el estudio de proyectos y actividades con base en la robótica educativa para el desarrollo curricular en diferentes niveles y establecer el grado de funcionalidad dependiendo de la etapa educativa y de la edad del alumnado. El trabajo en equipo, colaborativo, creativo y funcional que implican las actividades basadas en la robótica educativa puede ser un camino muy interesante en el trabajo por proyectos $\mathrm{y}$ competencial que requiere la didáctica del siglo XXI.

\section{9.- BIBLIOGRAFÍA}

Arandia, M., Alonso-Olea, M. J. \& Martínez-Domínguez, I. (2010). La metodología dialógica en las aulas universitarias. Revista de Educación, 352, 309-329.

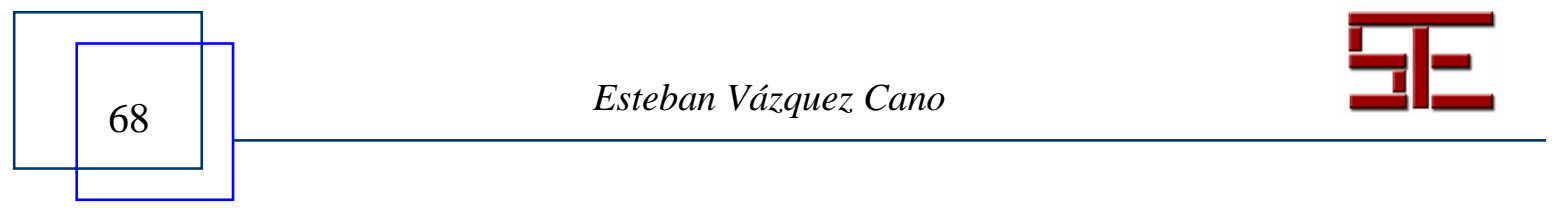




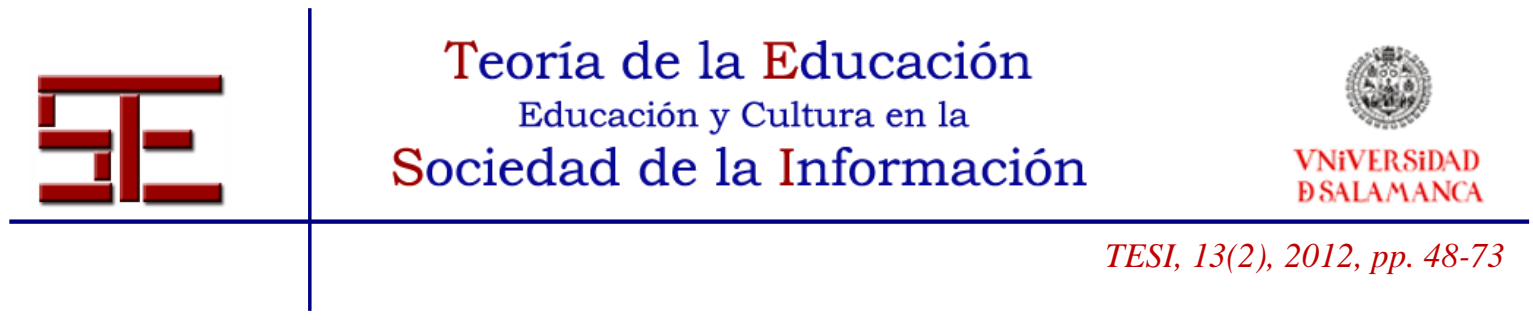

Bannan-Ritland, B., Dabbagh, N. \& Murphy, K. (2000). Learning Object Systems as Constructivist Learning Environments: Related Assumptions, Theories and Applications. En D. A. Wiley (Ed.), The Instructional Use of Learning Objects. Extraído el 1 de abril, 2012, de http://reusability.org/read/chapters/wiley.doc.

Blank, D. S., Kumar, D., Meeden, L. \& Yanco, H. (2004). Pyro: A python-based versatile programming environment for teaching robotics. Journal on Educational Resources in Computing (JERIC), Special Issue on robotics in undergraduate education, part I. 4 (3).

Blumenfeld, P. C., Soloway, E., Marx, R. W., Krajcik, J. S., Guzdial, M. \& Palincsar, A. (1991). Motivating project-based learning: Sustaining the doing, supporting the learning. Educational Psychologist, 26 (3-4).

Bolívar, A., Domingo, J. \& Fernández, M. (2001). La investigación biográficonarrativa en educación. Enfoque y metodología. Madrid: La Muralla.

Bransford, J. D. \& Stein, B. S. (1993). The Ideal Problem Solver. New York: Freeman.

Breazeal, C.L. (2002). Designing Sociable Robots (Intelligent Robotics and Autonomous Agents). Boston: Ed. Massachussets Institute of Technology.

Cabero, J. (2003). Principios pedagógicos, psicológicos y sociológicos del trabajo colaborativo: su proyección en la tele-enseñanza. En Martínez Sánchez, F. (Comp.). Redes de comunicación en la enseñanza (pp: 131-156). Barcelona: Paidós.

Carbonaro, M., Rex, M. \& Chambers, J. (2004). Using LEGO Robotics in a ProjectBased Learning Environment. The Interactive Multimedia Electronic Journal of Computer-Enhanced Learning, 6 (1).

Clandinin, D. J. \& Connelly, F. M. (1994). Personal experience methods. En N. K.

Coll, C. (2001). Constructivismo y educación: la concepción constructivista de la enseñanza y el aprendizaje. En C. Coll, J. Palacios y A. Marchesi (Comps.), Desarrollo psicológico y educación. 2. Psicología de la educación escolar. (pp: 157-188). Madrid: Alianza.

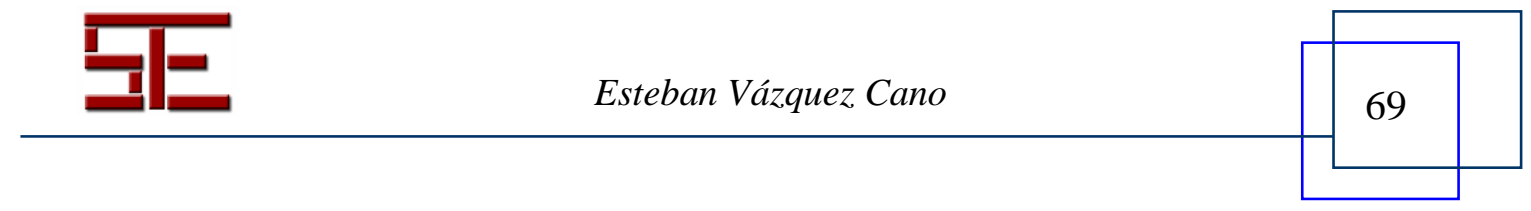




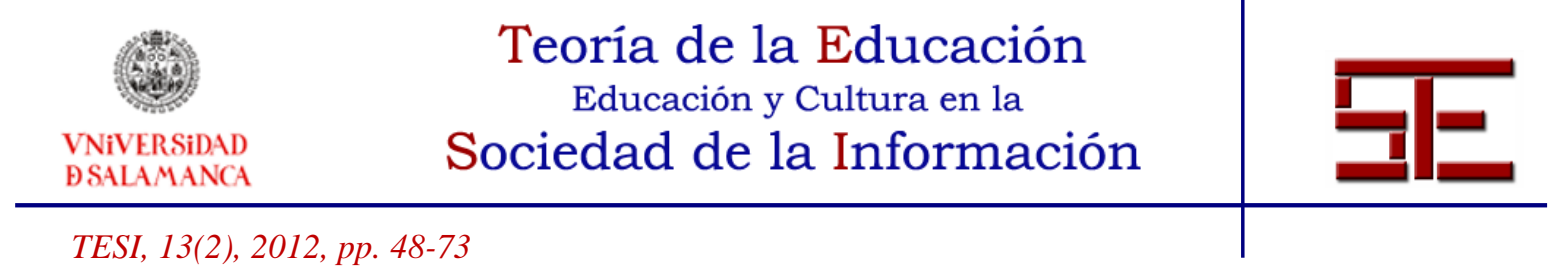

Denis, B. \& Hubert, S. (2001). Collaborative learning in an educational robotics environment. Computers in Human Behavior, 17, 465-480.

De Pablos, J., Colas, P. \& Villaciervos T. (2010). Políticas educativas, buenas prácticas y TIC en la comunidad autónoma andaluza. Teoría de la Educación. Educación y Cultura en la Sociedad de la Información, 11,1, 180-202.

Druin, A. \& Hendler, J. (2000). Robots for kids: Exploring new technologies for learning. San Diego, CA: Academic Press.

Erstad, O. (2002). Norwegian students using digital artefacts in project-based learning, Journal of Computer Assisted Learning, 18, 427-437.

Gagnon, G. W. \& Collay, M. (2001). Designing for Learning: Six Elements in Constructivist Classrooms. Corwin Press.

García-Valcárcel, A. (2009). Modelos y estrategias de enseñanza. Videoconferencia presentada como parte de los documentos de la materia "Modelos y estrategias de enseñanza”, de la Maestría en Educación del Instituto Tecnológico de Monterrey.

Han, S. \& Bhattacharya, K. (2001). Constructionism, Learning by Design, and Projectbased Learning. En M. Orey (Ed.), Emerging perspectives on learning, teaching, and technology. Extraído el 1 de abril, 2012, de http://www.coe.uga.edu/epltt/LearningbyDesign.htm.

Hernández, R., Fernández, C. \& Baptista, P. (2006). Metodología de la investigación. México: McGraw-Hill.

Hernández, F. \& Vergara, O. (2004). ¿Son útiles los diarios de la clase? Experiencia con el componente de investigación en el aula en la Universidad del Valle. Lenguaje, 32, 212-232.

Ionita, S. \& Ionita A. I. (2007). Robotics between "object" and "educational tool". Steps towards a constructionist methodological approach. En Proceedings of EcoMedia International Conference, 23-24 Nov. 2007, Pitesti, Romania. (pp: 119-125).

Jones, L. J. \& Flynn, A. N. (1993). Mobile robots: Inspiration to implementation. Wellesly, MA: A.K. Peters.

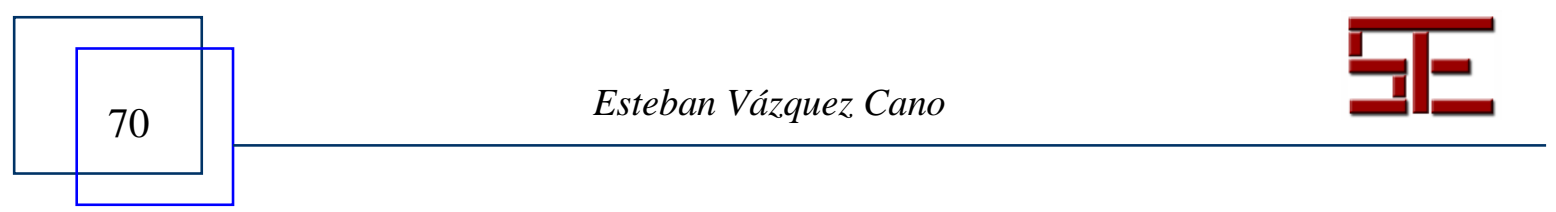




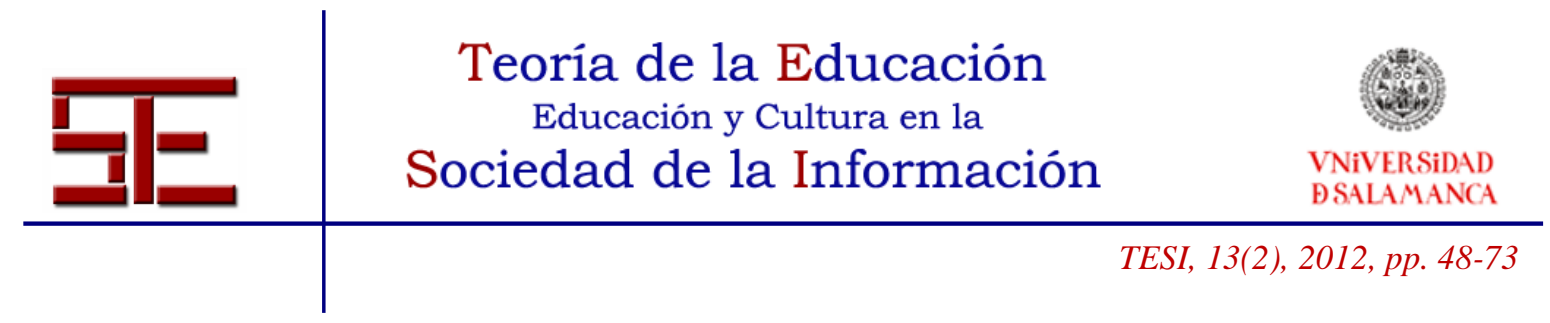

Knoll, M. (1997). The project method: Its vocational education origin and international development. Journal of Industrial Teacher Education, 34 (3), 59-80.

Kumar, D. (2004). Introduction to Special Issue on Robotics in Undergraduate Education. ACM Journal on Educational Resources in Computing, 4 (3).

Lipponen, L. (2003). Exploring foundations for computer supported collaborative learning. London: Routledge.

Martínez Miguélez, M. (2006). Validez y confiabilidad en la metodología cualitativa. Paradigma, 2, 7-34.

Mataric, M. (2004). Robotics Education for All Ages. En Proceedings, AAAI Spring Symposium on Accessible, Hands-on AI and Robotics Education. Palo Alto, CA, Mar 22-24, 2004.

Miglino, O., Lund, H. H. \& Cardaci, M. (1999). Robotics as an Educational Tool. Journal of Interactive Learning Research, 10:1, 25-48.

Mor, Y., Hoyles, C., Kahn, K., Noss, R. \& Simpson, G. (2006). Designing to see and share structure in number sequences. International Journal for Technology in Mathematics Education, 13, 65-78.

Oliver, R (2002). Learning settings and activities. H. Adelsberger, B. Collis, \& J. M. Pawlowski (Eds.) Handbook on information technologies for education and training. Berlin: Springer Verlag. (pp: 199-212).

Penner, D. E. (2001). Cognition, computers, and synthetic science: Building knowledge and meaning through modeling. En W. G. Secada (Ed.), Review of Research in Education (pp: 1-35). Washington, DC: American Educational Research Association.

Puntambekar, S. \& Kolodner, J. L. (2005). Toward implementing distributed scaffolding: Helping students learn science by design. Journal of Research in Science Teaching, 42 (2), 185-217.

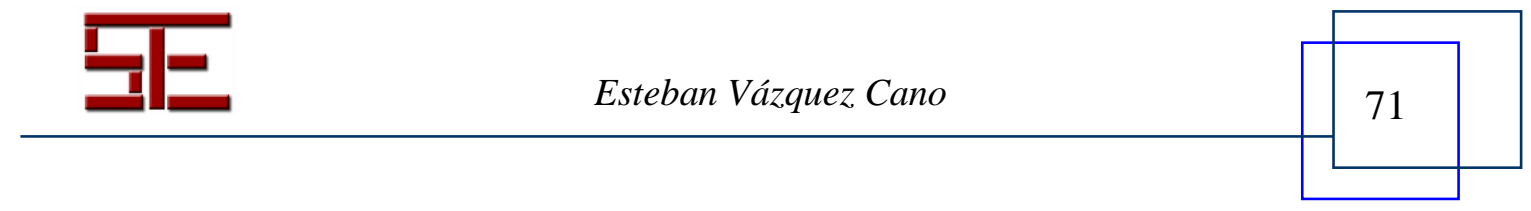




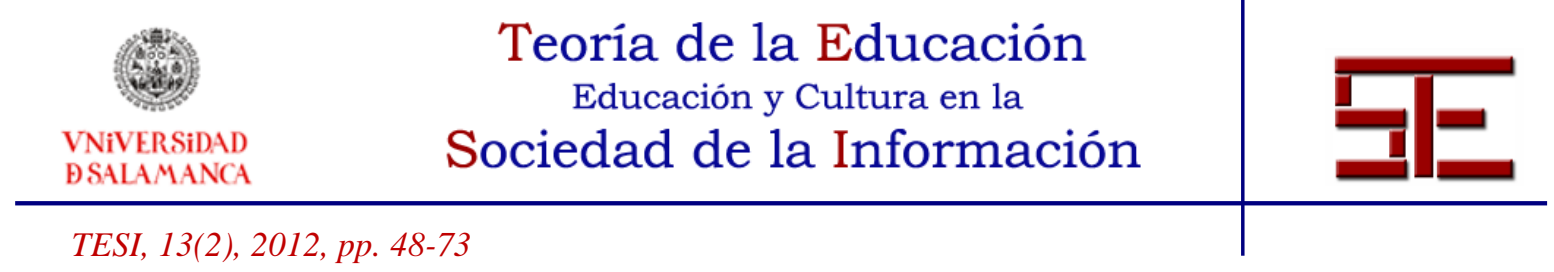

Resnick, M. (2006). Computer as Paintbrush: Technology, Play, and the Creative Society. En D. Singer, R. Golikoff, \& K. Hirsh-Pasek (Eds.), Play-Learning: How play motivates and enhances children's cognitive and social-emotional growth. Oxford University Press.

Resnick, M., Martin, F. G., Sargent, R. \& Silverman, B. (1996). Programmable bricks: Toys to think with. IBM Systems Journal, 35 (3-4), 443-452.

Schön, D. A. (1992). La formación de profesionales reflexivos. Hacia un nuevo diseño de la enseñanza y el aprendizaje en las profesiones. Barcelona: Paidós/M. E. C.

Sklar, E. \& Eguchi, A. (2004). Learning while Teaching Robotics. In AAAI Spring Symposium 2004 on Accessible Hands-on Artificial Intelligence and Robotics Education.

Sklar, E., Parsons, S. \& Azhar, M. Q. (2007). Robotics across the curriculum. En AAAI Spring Symposium on Robots and Robot Venues: Resources for AI Education. AAAI Press.

Thomas, J. W., Mergendoller, J. R. \& Michaelson, A. (1999). Project-based learning: A handbook for middle and high school teachers. Novato, CA: The Buck Institute for Education.

Vázquez Cano, E. \& Sevillano, Ma . L. (2011). Educadores en Red. Elaboración y edición de materiales audiovisuales para la enseñanza. Madrid: UNED-Ediciones Académicas.

Weiss, R. I. (2008). Overcast. Finding Your Bot-Mate: Criteria for Evaluating Robot Kits for Use in Undergraduate Computer Science Education. Journal of Computing Sciences in Colleges, 24 (2), 43-49.

\section{Referencias legislativas}

DECRETO 69/2007. Decreto 69 /2007, de 28 de mayo, por el que se establece y ordena el currículo de la educación secundaria obligatoria en la comunidad autónoma de Castilla-La Mancha.

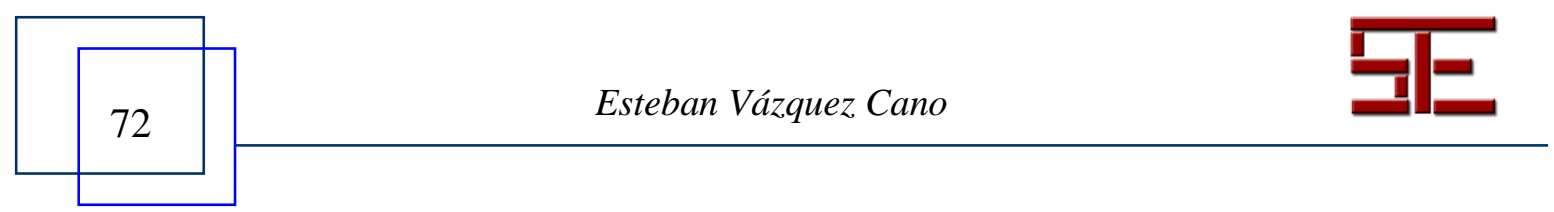




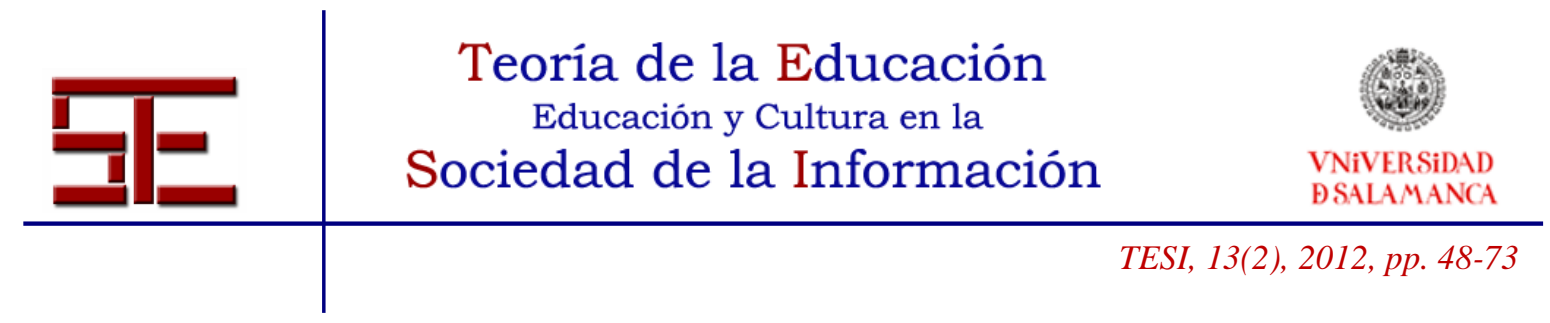

OECD (2002). Definition and Selection of Competencies (DeSeCo): Theoretical and Conceptual Foundations: Strategy Paper.

LOE (2006). Ley Orgánica 2/2006, de 3 de mayo, de Educación.

MEC (2006). Currículo y competencias básicas. Documento de trabajo.

OFFICIAL JOURNAL OF THE EUROPEAN UNION (2006). Recommendation of the European Parliament and of the Council of 18 December 2006 on key competences for lifelong learning. (L394/14- 2006/962/CE).

Para citar el presente artículo puede utilizar la siguiente referencia:

Vázquez Cano, E. (2012). Simulación robótica con herramientas 2.0 para el desarrollo de competencias básicas en ESO. Un estudio de casos. Revista Teoría de la Educación: Educación y Cultura en la Sociedad de la Información. 13(2), 48-73 [Fecha de consulta: $\mathrm{dd} / \mathrm{mm} / \mathrm{aaaa}]$.

http://campus.usal.es/ revistas_trabajo/index.php/revistatesi/article/view/8999/9244

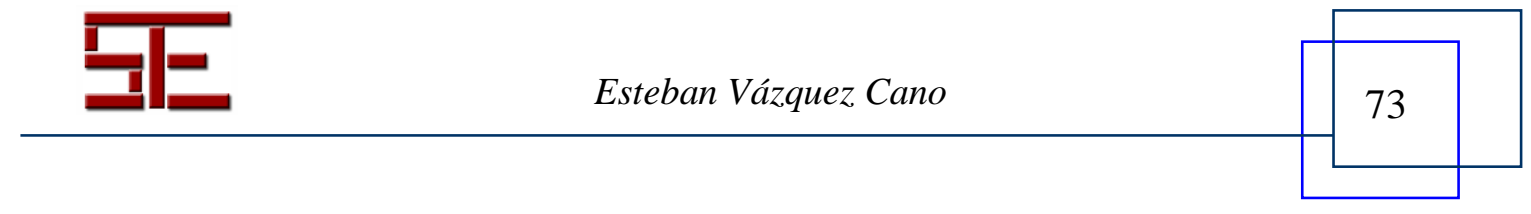

